ARTICLE

https://doi.org/10.1038/s41467-019-11378-4

\title{
The Eleanor ncRNAs activate the topological domain of the ESR1 locus to balance against apoptosis
}

\author{
Mohamed Osama Ali Abdalla (10 1,2,8, Tatsuro Yamamoto1,3,4,8, Kazumitsu Maehara5, Jumpei Nogami ${ }^{5}$, \\ Yasuyuki Ohkawa ${ }^{5}$, Hisashi Miura $\mathbb{1 0}^{6}{ }^{6}$, Rawin Poonperm ${ }^{6}$, Ichiro Hiratani ${ }^{6}$, Hideki Nakayama ${ }^{4}$, \\ Mitsuyoshi Nakao ${ }^{1,7,9}$ \& Noriko Saitoh ${ }^{1,3,9}$
}

MCF7 cells acquire estrogen-independent proliferation after long-term estrogen deprivation (LTED), which recapitulates endocrine therapy resistance. LTED cells can become primed for apoptosis, but the underlying mechanism is largely unknown. We previously reported that Eleanor non-coding RNAs (ncRNAs) upregulate the ESR1 gene in LTED cells. Here, we show that Eleanors delineate the topologically associating domain (TAD) of the ESR1 locus in the active nuclear compartment of LTED cells. The TAD interacts with another transcriptionally active TAD, which is $42.9 \mathrm{Mb}$ away from ESR1 and contains a gene encoding the apoptotic transcription factor $\mathrm{FOXO3.} \mathrm{Inhibition} \mathrm{of} \mathrm{a} \mathrm{promoter-associated} \mathrm{Eleanor} \mathrm{suppresses} \mathrm{all} \mathrm{genes}$ inside the Eleanor TAD and the long-range interaction between the two TADs, but keeps FOXO3 active to facilitate apoptosis in LTED cells. These data indicate a role of ncRNAs in chromatin domain regulation, which may underlie the apoptosis-prone nature of therapyresistant breast cancer cells and could be good therapeutic targets.

\footnotetext{
${ }^{1}$ Department of Medical Cell Biology, Institute of Molecular Embryology and Genetics, Kumamoto University, Kumamoto 860-0811, Japan.

2 Department of Clinical Pathology, Faculty of Medicine, Suez Canal University, Ismailia 41522, Egypt. ${ }^{3}$ Division of Cancer Biology, The Cancer Institute of JFCR, Tokyo 135-8550, Japan. ${ }^{4}$ Department of Oral and Maxillofacial Surgery, Faculty of Life Sciences, Kumamoto University, Kumamoto 860-8556, Japan. ${ }^{5}$ Division of Transcriptomics, Medical Institute of Bioregulation, Kyushu University, Fukuoka 812-8582, Japan. ${ }^{6}$ Laboratory for Developmental Epigenetics, RIKEN Center for Biosystems Dynamics Research (BDR), Kobe 650-0047, Japan. ${ }^{7}$ Core Research for Evolutional Science and Technology (CREST), Japan Science and Technology Agency, Tokyo 104-0004, Japan. ${ }^{8}$ These authors contributed equally: Mohamed Osama Ali Abdalla, Tatsuro Yamamoto. ${ }^{9}$ These authors jointly supervised this work: Mitsuyoshi Nakao, Noriko Saitoh. Correspondence and requests for materials should be addressed to M.N. (email: mnakao@gpo.kumamoto-u.ac.jp) or to N.S. (email: noriko.saito@jfcr.or.jp)
} 
B reast cancers expressing estrogen receptor-a (ER) depend on estrogen for their growth. ER-positive cancer patients are treated with endocrine therapies that function by blocking estrogen production; these therapies include the use of aromatase inhibitors (AIs) ${ }^{1}$. After treatment, the disease frequently recurs because cancer cells gradually develop tolerance to estrogen deprivation ${ }^{1-3}$.

Long-term estrogen deprivation (LTED) cells are established as survivors, after culturing MCF7 ER-positive human breast cancer cells in an estrogen-depleted medium for a long duration ${ }^{4-7}$. LTED cells therefore represent the development of AI resistance or postmenopausal tumorigenesis. Several studies, including the present study, have shown that LTED cells are primed for cell death, which may be triggered by several polyphenols ${ }^{8,9}$. High estrogen concentrations may have a similar effect; it has been shown to be effective against AI-resistant breast cancers, although the treatment is seemingly paradoxical ${ }^{10,11}$. The mechanism underlying LTED cell death may be related to epigenetic chromatin structure, but details of this are largely unknown.

In the nucleus, chromosomes form higher-order structures that govern gene regulation ${ }^{12,13}$. To analyze inter-chromosomal interactions, C-techniques have been developed and shed light on the three-dimensional (3D) genome structures within the nucleus. The original Hi-C analysis classified the chromosome organization into two patterns: transcriptionally active $\mathrm{A}$ and inactive $\mathrm{B}$ compartments ${ }^{12}$. Subsequent higher-resolution analyses showed that neighboring chromosomal regions were folded into self-associating topologically associating domains (TADs) that are several hundred $\mathrm{kb}$ to $1-2 \mathrm{Mb}$ in size ${ }^{14,15}$. In addition, intense point-to-point loops have been detected by highresolution $\mathrm{Hi}-\mathrm{C}$, and some are anchored with the chromatin protein, $\mathrm{CTCF}^{16}$.

An important recent observation is that the majority of the genome is continuously transcribed into non-coding RNAs (ncRNAs), many of which are specifically transcribed according to cell type or disease ${ }^{17}$. Among them are various types of long ncRNAs including those resulting from divergent transcription at the transcription start sites (TSSs) of protein-coding genes ${ }^{18-20}$. These promoter-associated ncRNAs (paRNAs) exist throughout the genome and positively correlate with proximal gene activities.

Several possibilities of ncRNA-mediated chromatin domain regulation have been suggested ${ }^{13,21}$. ncRNAs that are associated with proteins critical for chromatin domain boundaries, including CTCF and cohesin components, have been identified ${ }^{22}$. ncRNAs that create large RNA foci or clouds in the nucleus regulate gene expression by chromatin domain units ${ }^{23,24}$. Bioinformatics analyses have identified over 700 ncRNA loci that overlap with TAD borders ${ }^{25}$. These results suggest that ncRNAs could be potential therapeutic target candidates ${ }^{26}$. However, the involvement of ncRNAs in chromatin domain regulation in cancer cell adaptation has not been demonstrated yet.

Previously, we and others showed that the gene encoding ER (ESR1), to which cancer cells are transcriptionally addicted, is critically upregulated during LTED adaptation ${ }^{4,27}$. We showed that, in LTED cells, a cluster of ncRNAs, ESR1 locus enhancing and activating non-coding RNAs (Eleanors) were transcribed from a large chromatin domain containing the ESR1 locus and then remained at the transcriptionally active ESR1 locus to form RNA clouds and cis-activate transcription ${ }^{27}$. Eleanors were found to be effectively suppressed with resveratrol due to its estrogenic effect. Eleanors were present in ER-positive breast cancer tissues. However, the mechanisms and significance of the Eleanormediated gene and chromatin regulation remained to be investigated.

In the present study, we performed a 4C-Seq (chromosome conformation capture combined with high-throughput sequencing) analysis using the ESR 1 promoter as bait and found that Eleanors delineate the TAD of the ESR1 locus. We also found that the identified promoter-associated Eleanor mediates longrange chromatin interaction between the ESR1 and FOXO3 loci, which function in cell proliferation and apoptosis, respectively. Inhibition of Eleanor disrupted the long-range chromatin interaction and suppressed ESR1, but not FOXO3, leading cells to apoptosis. Our observations provide an epigenetic model for the seemingly paradoxical upregulation of the two genes and may explain the apoptosis priming of breast cancer cells undergoing therapeutic estrogen deprivation.

\section{Results}

Identification of the Eleanor TAD on human chromosome 6q25.1. To explore the dynamics of higher-order chromosomal organization in breast cancer cells, we used three cellular models: MCF7, LTED, and LTED-RES cells (Fig. 1a). MCF7 cells represent human ER-positive breast cancer. LTED cells were established by culturing MCF7 cells in an estrogen-depleted medium over a long duration ( $>3$ months). At an early stage of estrogen deprivation, cell death occurs because MCF7 cells require estrogen for growth. Those that survive are known as LTED cells and represent breast cancer that has acquired resistance to endocrine therapy ${ }^{4,28}$. To obtain LTED-RES cells, LTED cells were treated with $100 \mu \mathrm{M}$ resveratrol for $24 \mathrm{~h}$. LTED-RES cells also undergo cell death that could recapitulate estrogen additive therapy because resveratrol and estrogen are structurally related. Previously, we showed that Eleanor nuclear ncRNAs emerged from an approximately $700 \mathrm{~kb}$ chromatin region including the ESR1 locus to upregulate $E S R 1^{27}$. The resveratrol treatment repressed Eleanors and downregulated ESR1 expression ${ }^{27}$.

To investigate the 3D genomic structures of Eleanor-expressing chromosomal regions, we performed 4C-Seq analysis of MCF7 and LTED cells using the TSS of ESR1 as bait. We designed two 4C-Seq sets, one using DpnII (exp-A) and another using HindIII (exp-B) for the first restriction enzyme digestions of the fixed nuclear chromosomes (Supplementary Fig. 1a). The resultant circular DNAs after ligation, which contained genomic sequences fused with the bait, were sequenced and their reproducibility was confirmed between the replicated experiments as well as experiments using different restriction enzymes (Supplementary Fig. 1b, c).

As expected from the nature of $\mathrm{C}$-technology, massive peaks were detected around the bait site (Fig. 1b). Sharp transitions occurred at chr6:151,650,000-151,750,000 and 152,650,000-152,750,000, and their $4 \mathrm{C}$-Seq reads were statistically distinct from those of the neighboring regions $(P<0.05)$, according to Turkey-Kramer test along chr6: 151,000,000-153,000,000 with $100-\mathrm{kb}$ windows (Supplementary Fig. 2a). We found that the sharp transitions coincided with the TAD boundaries determined by a previous $\mathrm{Hi}-\mathrm{C}$ study using MCF7 cells (yellow vertical lines in Fig. 1b) ${ }^{29}$, as well as those from other $\mathrm{Hi}-\mathrm{C}$ data of different cell types (yellow vertical lines in Supplementary Fig. $2 b, c)$. The alignment of this TAD and our previous RNA-Seq data ${ }^{27}$ revealed that the TAD coincided with the Eleanor-expressing chromatin domain (chr6: 151,750,000-152,750,000) (Fig. 1b). Therefore, the Eleanor TAD resides at 6 q25.1 on human chromosome 6 exists in both MCF7 and LTED cells and contains ESR1 and 3 other genes: CCDC170, C6orf211, and RMND1 (Fig. 1b).

To investigate the significance of the Eleanor TAD, we measured the transcriptional activities of genes both within and outside the Eleanor TAD. Quantitative reverse transcription polymerase chain reaction (qRT-PCR) showed that only the genes within the TAD (RMND1, C6orf211, CCDC170, and ESR1) were co-upregulated in LTED cells and cooperatively decreased 
a

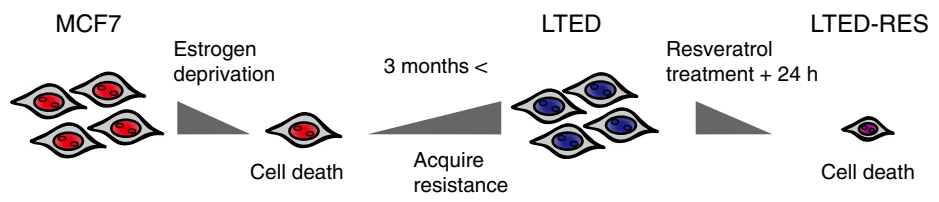

b
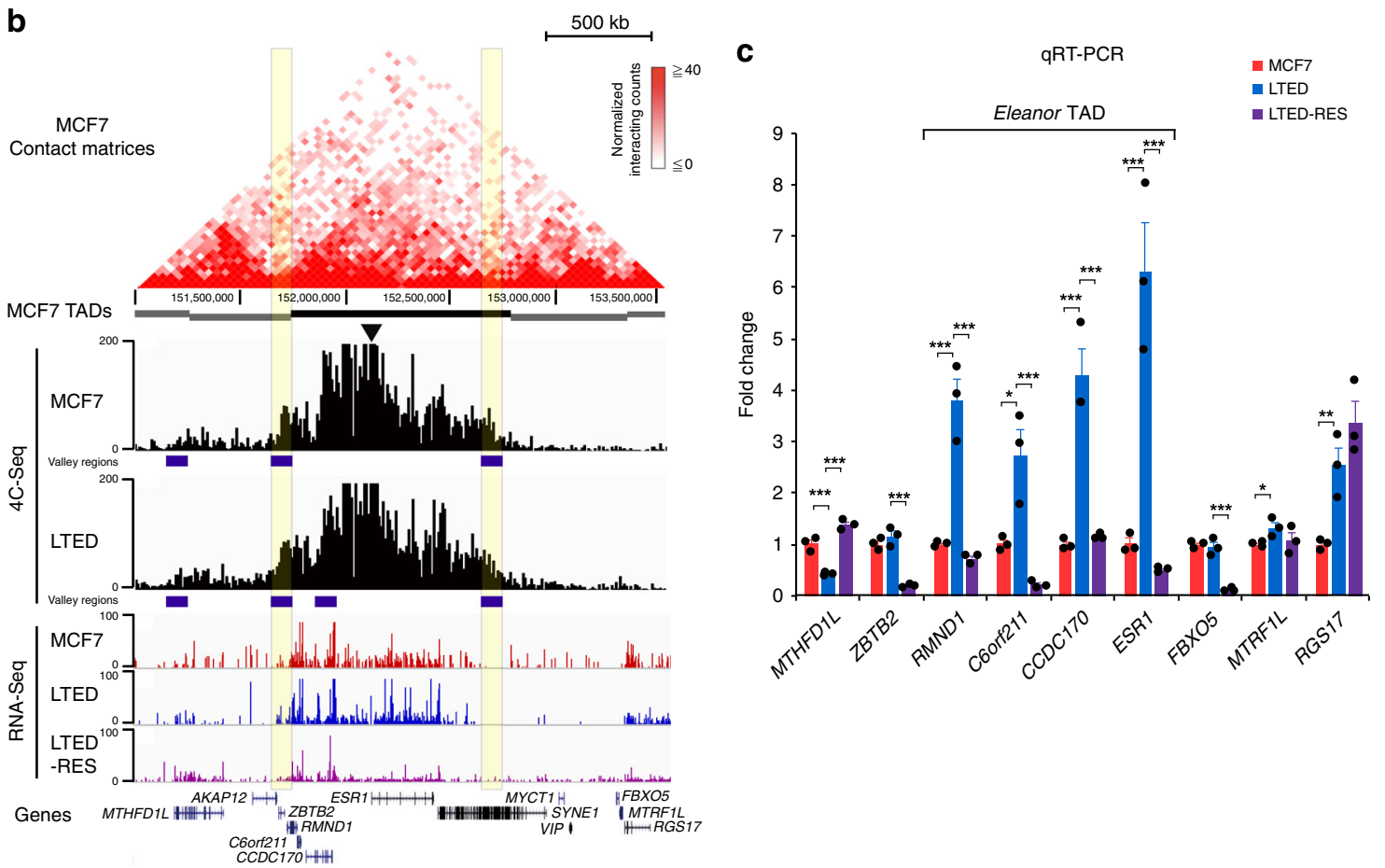

MCF7
Contact matrices
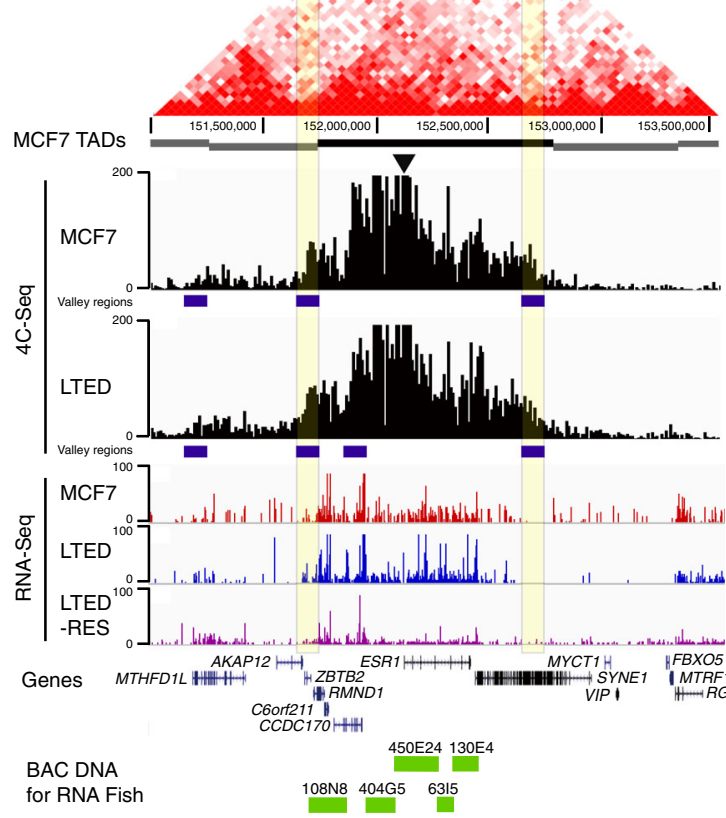

d
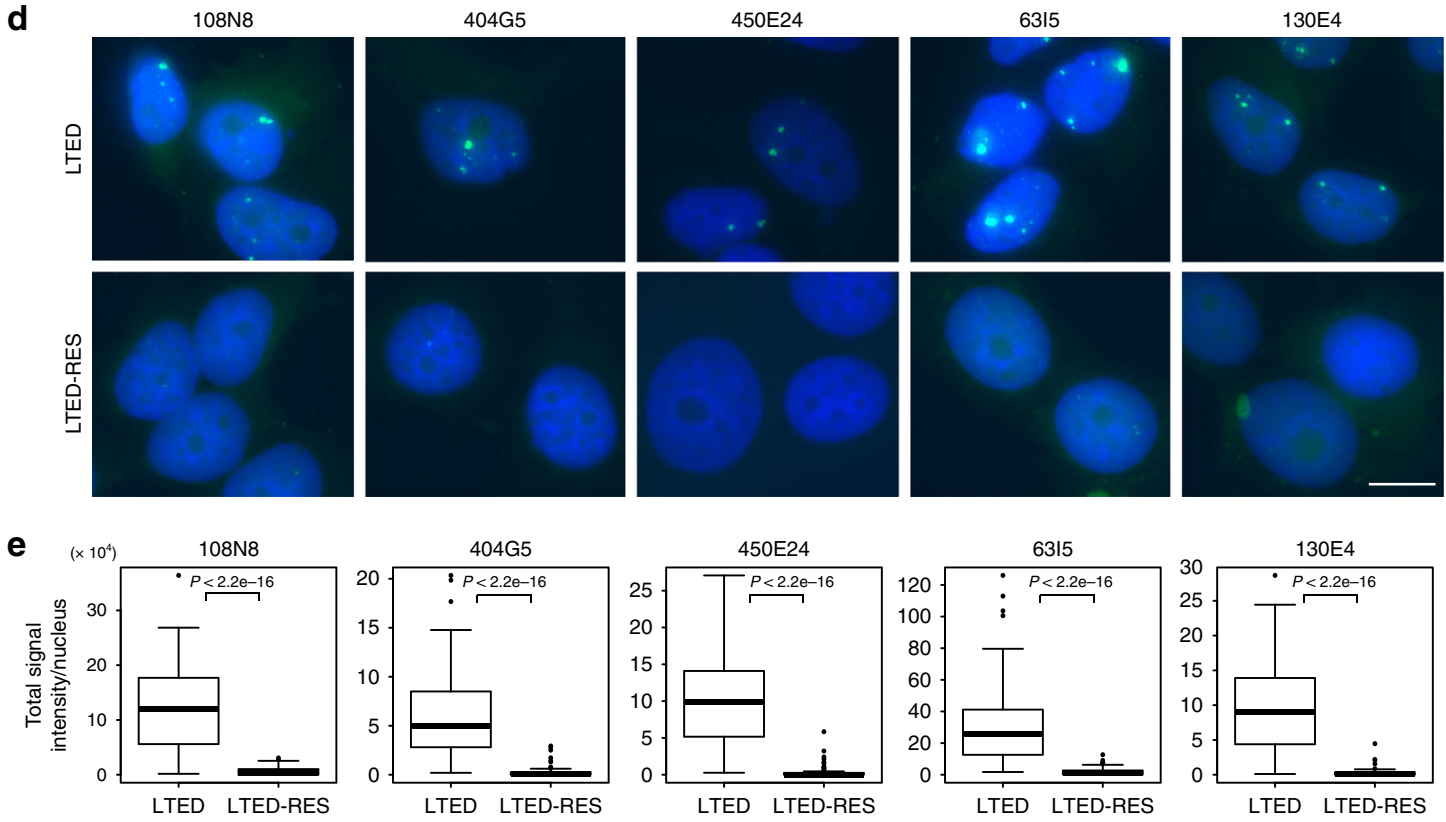

after resveratrol treatment (Fig. 1c). The same TAD-based gene regulation was observed in another ER-positive breast cancer cell line HCC1428 as well as in LTED cells (HCC1428-LTED) and resveratrol-treated cells (HCC1428-LTED-RES) created from the cell line (Supplementary Fig. 2d). Our RNA-Seq of MCF7, LTED, and LTED-RES cell data also support the theory that the four clustered genes are co-regulated in the Eleanor TAD
(Supplementary Table 1$)^{27}$. Consistently, the active form of RNA polymerase II was bound to all four genes in the LTED cells, and the binding was reduced in LTED-RES cells (Supplementary Fig. 2e).

Eleanors, clusters of ncRNAs that are transcribed from intergenic regions of the Eleanor TAD form larger RNA clouds in LTED nuclei, compared with those in MCF7 nuclei ${ }^{27}$. Our 
Fig. 1 Eleanor topologically associating domain (TAD) corresponds to the Eleanor-expressing chromatin domain. a Cells used in this study: MCF7, long-term estrogen deprivation (LTED), and LTED-RES cells. MCF7 cells were cultured in estrogen-deprived medium for $>3$ months to establish LTED cells, which was then treated with $100 \mu \mathrm{M}$ resveratrol for $24 \mathrm{~h}$ (LTED-RES cells). Cell death occurs early during estrogen deprivation and after resveratrol treatment. b Alignment of TADs and $4 \mathrm{C}$-Seq (chromosome conformation capture combined with high-throughput sequencing) profiles in the region, including the ESR1 gene on human chromosome 6 (6q25.1). Top: Hi-C contact matrix and predicted TAD positions (gray and black bars) ${ }^{29}$. Middle: 4C-Seq (this study) and RNA-Seq ${ }^{27}$ profiles of the indicated cells. The arrowhead indicates the position of the $4 \mathrm{C}$ bait, and the dark blue bars indicate the valley regions of the $4 C$ peaks (Supplementary Fig. 2a). Bottom: positions of RefSeq genes and BAC clones (green bars) used as probes for RNA fluorescence in situ hybridization (FISH) in this study. The black bar TAD with yellow highlights delineates the position of the Eleanor TAD. c Quantitative reverse transcription polymerase chain reaction analysis for the expression levels of genes inside and outside the Eleanor TAD. Genes inside the Eleanor TAD were cooperatively activated in LTED cells and were downregulated by resveratrol treatment (LTED-RES). The value of MCF7 expression level was set to 1. Data are representative of three independent experiments (mean \pm s.e.m.). $P$ values were calculated using unpaired, two-tailed, Student's $t$ test $\left({ }^{\star} P<0.05\right.$, ${ }^{\star \star} P<$ $\left.0.01,{ }^{\star \star \star} P<0.001\right)$. d RNA FISH analysis along the Eleanor TAD. BAC clones used as probes are indicated above each panel. Eleanor RNA foci were diminished with resveratrol treatment (LTED-RES). Scale bar, $10 \mu \mathrm{m}$. e Quantification of RNA FISH. $n=50-100$ nuclei per sample. Boxplots shown are center line, median; box limits, upper and lower quartiles; whiskers, $1.5 \times$ interquartile range; points, outliers. $P$ values were calculated using two-tailed, Mann-Whitney $U$ test

RNA fluorescence in situ hybridization (FISH) analysis used a series of probes covering the entire Eleanor TAD region to show that the cloud was diminished by resveratrol treatment (Fig. 1d, e). These results indicated that resveratrol treatment affects the transcription of all genes in the TAD possibly mediated by Eleanors.

Interaction of the ESR1 promoter with the FOXO3 gene locus. Upon further analysis of our 4C-Seq data, we observed intrachromosomal ESR1 promoter interactions along the entirety of chromosome 6 in MCF7 and LTED cells (Fig. 2a and Supplementary Fig. 3a). Alignment of the 4C-Seq with MCF7 cells A/B compartment profiles ${ }^{29}$ revealed that the Eleanor TAD was in the A compartment (Fig. 2a and Supplementary Fig. 3a). 4C-Seq peaks representing interactions with the ESR1 promoter were prominent near the bait region and gradually reduced in intensity with increased distance from the bait, as expected. However, the 4C peaks tended to coincide with the A compartments throughout the chromosome. In total, $66 \%$ and $72 \%$ of the regions with $4 \mathrm{C}$ peaks overlapped with the A compartments in MCF7 and LTED cells, respectively (Supplementary Fig. 3b). It suggests that the ESR1 promoter region in the Eleanor TAD interacted with genes in the A compartments of multiple regions of chromosome 6. Consistently, ESR1 promoter-interacting regions showed moderate coincidence with genes that were upregulated in LTED cells (red bars below 4C-Seq profiles in Fig. 2a and Supplementary Fig. 3a). In total, $60 \%$ of the regions with $4 \mathrm{C}$ peaks in the entire chromosome 6 overlapped with the genes upregulated in LTED cells (Supplementary Fig. 3c).

We then focused on one of the ESR1 promoter-interacting regions, $6 \mathrm{q} 21$, which is approximately $42.9 \mathrm{Mb}$ away from the ESR1 promoter. First, we analyzed the interaction between the ESR1 promoter and regions inside (b site) and outside (d site) of $6 \mathrm{q} 21$ using DNA FISH (Fig. 2b, c). The frequency of overlapping signals was high at the $b$ site, but not at the $d$ site, in LTED cells (Fig. 2b, c and Supplementary Fig. 3d). Furthermore, 3C-quantitative PCR (3C-qPCR) confirmed that the level of interaction between the ESR 1 promoter and the $\mathrm{b}$ site was high in LTED cells, but not at the a, c, or d sites (Fig. 2d). Using genomic qPCR, we confirmed that the increased interaction frequency of the ESR1 promoter-b site was not due to genome amplification because there was no detectable genome amplification at sites a-d, as with the ESR1 promoter (Supplementary Fig. 3e).

To understand the significance of genome interaction between the ESR1 promoter and $6 \mathrm{q} 21$, we investigated transcriptional activities at the b site of $6 \mathrm{q} 21$. Our qRT-PCR and RNA-Seq showed that most genes at the $\mathrm{b}$ site were also upregulated in LTED cells derived from MCF7 (Fig. 2e, Supplementary Table 2) and HCC1428 cells (Supplementary Fig. 3f). This suggested that, although the two domains are located over $40 \mathrm{Mb}$ apart on chromosome 6 , they physically associate in the nuclear space and are co-upregulated in LTED cells.

One of the genes at the b site was FOXO3, which belongs to the $O$ subclass of the forkhead family of transcription factors, implicated in the induction of apoptosis via upregulation of genes necessary for cell death such as Bim and PUMA or downregulation of anti-apoptotic proteins, such as $F L I P^{30,31}$. Although the interaction between genes for growth (ESR1) and apoptosis (FOXO3) appeared contradictory at first, apoptotic activity is frequently enhanced in cancer, both in cultured cell lines and patient cells ${ }^{6,8,32,33}$. During LTED cell establishment, a massive number of cells die immediately after estrogen deprivation commences. Therefore, FOXO3 may play an important role in cellular adaptation to this environmental change.

To test whether upregulation of FOXO3 is important for cell death during LTED cell establishment, we knocked down FOXO3 at an early stage (2 months) of estrogen deprivation and measured cell growth, vitality, and apoptosis (Supplementary Fig. $4 \mathrm{a}-\mathrm{d}$ ). We found that cell death was suppressed by downregulating $\mathrm{FOXO3}$, suggesting that $\mathrm{FOXO} 3$ is functionally activated during estrogen deprivation. In addition, we overexpressed FOXO3 in LTED cells and found that it induced cell death (Supplementary Fig. 4e-g). Knocking down another coupregulated but irrelevant gene, $S N X 3$, did not induce cell apoptosis (Supplementary Fig. 4h, i). Altogether, FOXO3 is at least partly responsible for cell death during estrogen deprivation, and the surviving LTED cells may overcome its apoptosisinducing effect, probably by acquiring an ability to upregulate the proliferation gene ESR $1^{27}$.

Resveratrol suppressed the interaction between ESR1 and FOXO3. To investigate whether the chromatin interaction of the ESR1 promoter was mediated by Eleanors, we inhibited Eleanors with resveratrol in LTED cells and performed the $\mathrm{Hi}-\mathrm{C}$ analysis. A comparison of the normalized contact matrices for LTED and LTED-RES showed no dramatic Mb-sized changes (Fig. 3a and Supplementary Fig. 5a). However, local changes were enriched in the A compartments ( $\log _{2}$ fold changes in contact (LTED/LTEDRES), shown as blue and red bars below the contact matrix in Fig. 3a), and one was found at the FOXO3 gene (b site in Fig. 3a). These suggest that the contact between the ESR1 promoter and FOXO3 became unstable with resveratrol treatment. Our 3CqPCR showed that the ESR1 promoter and the b site were dissociated after resveratrol treatment (Fig. 3b and Supplementary Fig. 5). These results suggest the Eleanor-mediated long-range chromatin interaction. 
a
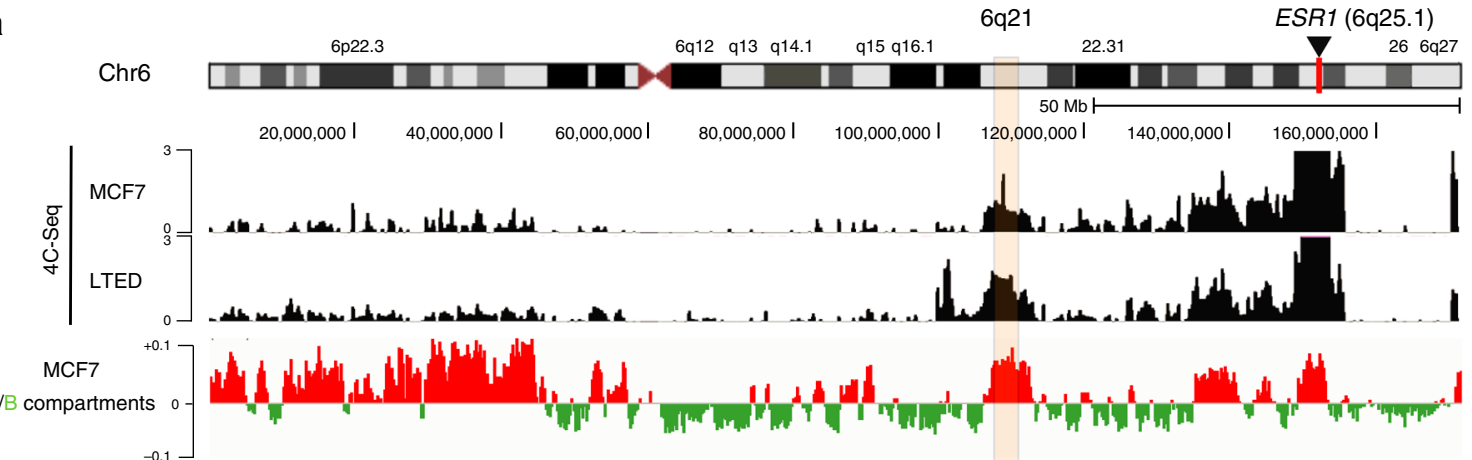

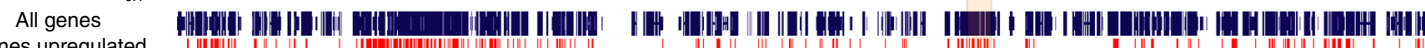

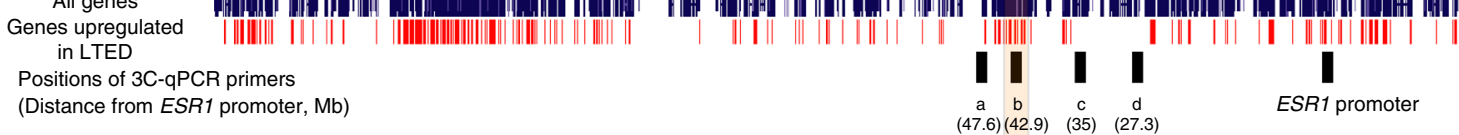

BAC DNA

for DNA FISH

(47.6) (42.9) (35) (27.3)

b-BAC d-BAC

p-BAC

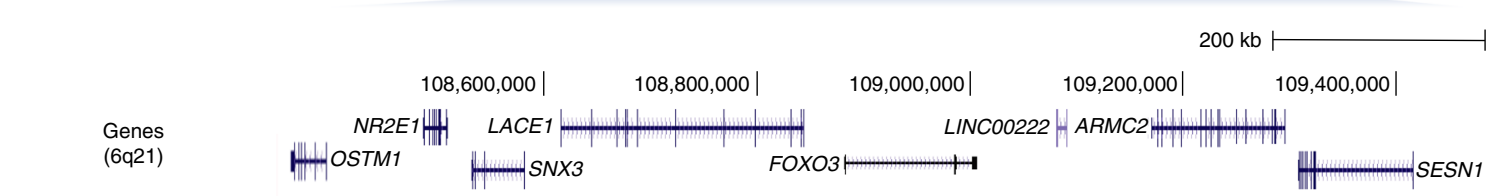

b
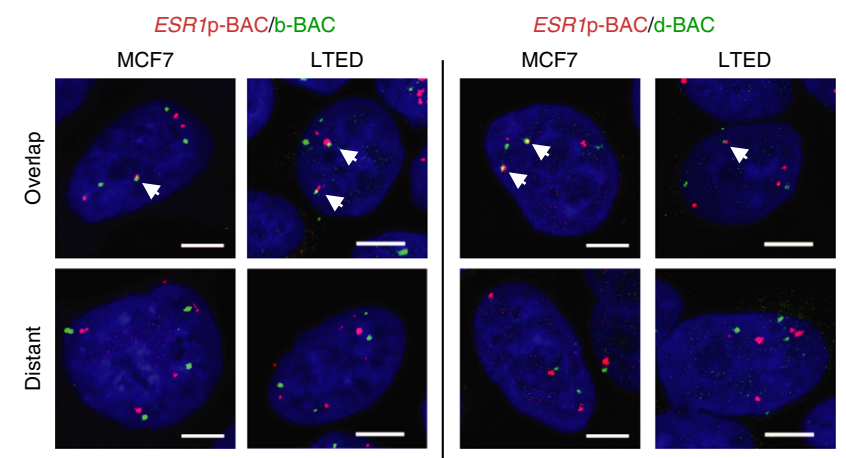

\#Nucle
analyzed

232

136

235

C

d

$3 C-q P C R$

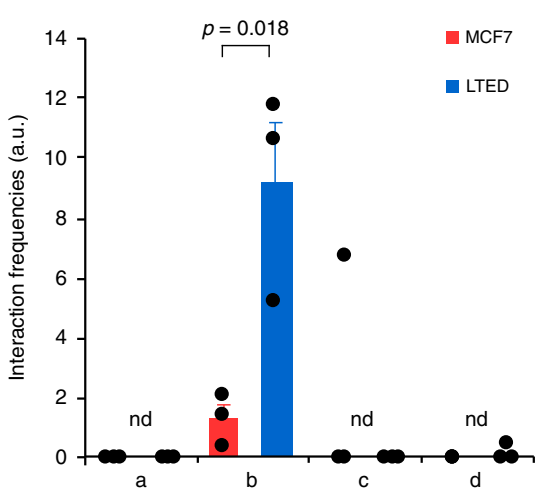

It should be noted that the FOXO3 gene remained upregulated in LTED-RES (Fig. $3 c$ and Supplementary Fig. 5c), in good agreement with the fact that LTED cells undergo apoptosis upon resveratrol treatment ${ }^{34}$. The expression levels of other genes in 6q21, SNX, and LACE1 were reduced (Fig. 3c, Supplementary Fig. 5c, and Supplementary Table 2). This is not due to RNA stability, because the FOXO3 RNA was even less stable than

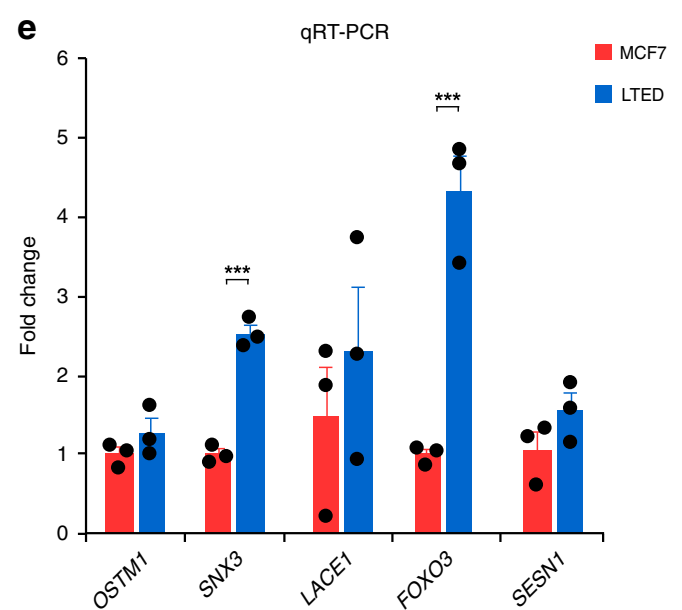

others (Supplementary Fig. 6a). Furthermore, the binding of the active form of RNA polymerase II to the $\mathrm{FOXO3}$ gene was maintained in LTED-RES cells (Supplementary Fig. 5d).

There are 15 other apoptotic genes that were co-regulated with FOXO3. They were upregulated in LTED cells, and their high expression was maintained in LTED-RES cells. (Supplementary Fig. 6b, Supplementary Table 3). Among them, three genes were 
Fig. 2 4C-Seq (chromosome conformation capture combined with high-throughput sequencing) analysis revealed a long-range interaction between ESR1 and FOXO3 genes in long-term estrogen deprivation (LTED) cells. a Alignment of 4C-Seq and A/B compartment profiles along the entirety of human chromosome 6 (UCSC genome browser, hg19 assembly). Top: 4C-Seq data showing intra-chromosomal interactions with the ESR1 promoter used as bait (arrowhead). Middle: Distribution of A (red) and B (green) compartments in MCF7 cells ${ }^{29}$. Note the positive correlation relationship between black peaks from 4C-Seq and red peaks representing A compartments. Bottom: distribution of all genes (blue) and genes upregulated in LTED cells (red) ${ }^{27}$. Thick blue bars represent the positions of 3C-qPCR (chromosome conformation capture-quantitative polymerase chain reaction) primers (a-d and the ESR1 promoter) used in this study. Green and red bars represent BAC DNA used as DNA fluorescence in situ hybridization (FISH) probes in b, c. Bottom: RefSeq genes at the $b$ site. It should be noted that the 4C-Seq profiles showed interaction peaks between b site at 6q21 and the ESR1 promoter (bait for 4C). b Representative DNA FISH images of the ESR1 locus (red, using ESR1p-BAC probe) and b and d sites (green, using b-BAC and d-BAC probes, respectively). The position of each probe is shown in a (green and red bars). Scale bar, $5 \mu \mathrm{m}$. c Quantification of the DNA FISH analysis in $\mathbf{b}$. Nuclei with overlapping signals at the ESR1-b site or ESR1-d site were measured. The frequencies of interaction between the ESR1 and $b$ site increased in LTED cells. $P$ values were calculated using two-tailed Fisher's exact test. d 3Cq-PCR analysis shows interaction frequencies between the ESR1 promoter and a-d sites in MCF7 and LTED cells. Positions of sites a-d are shown in a (blue bars). e Relative expression levels of genes at 6q21 in MCF7 and LTED cells. The MCF7 expression level was set to 1. Data presented in $\mathbf{d}$, e are representative of three independent experiments (mean \pm s.e.m.). $P$ values were calculated using unpaired, two-tailed, Student's $t$ test $\left({ }^{\star \star \star} P<0.001\right.$, n.d. $=$ not detected in at least one experiment)

a

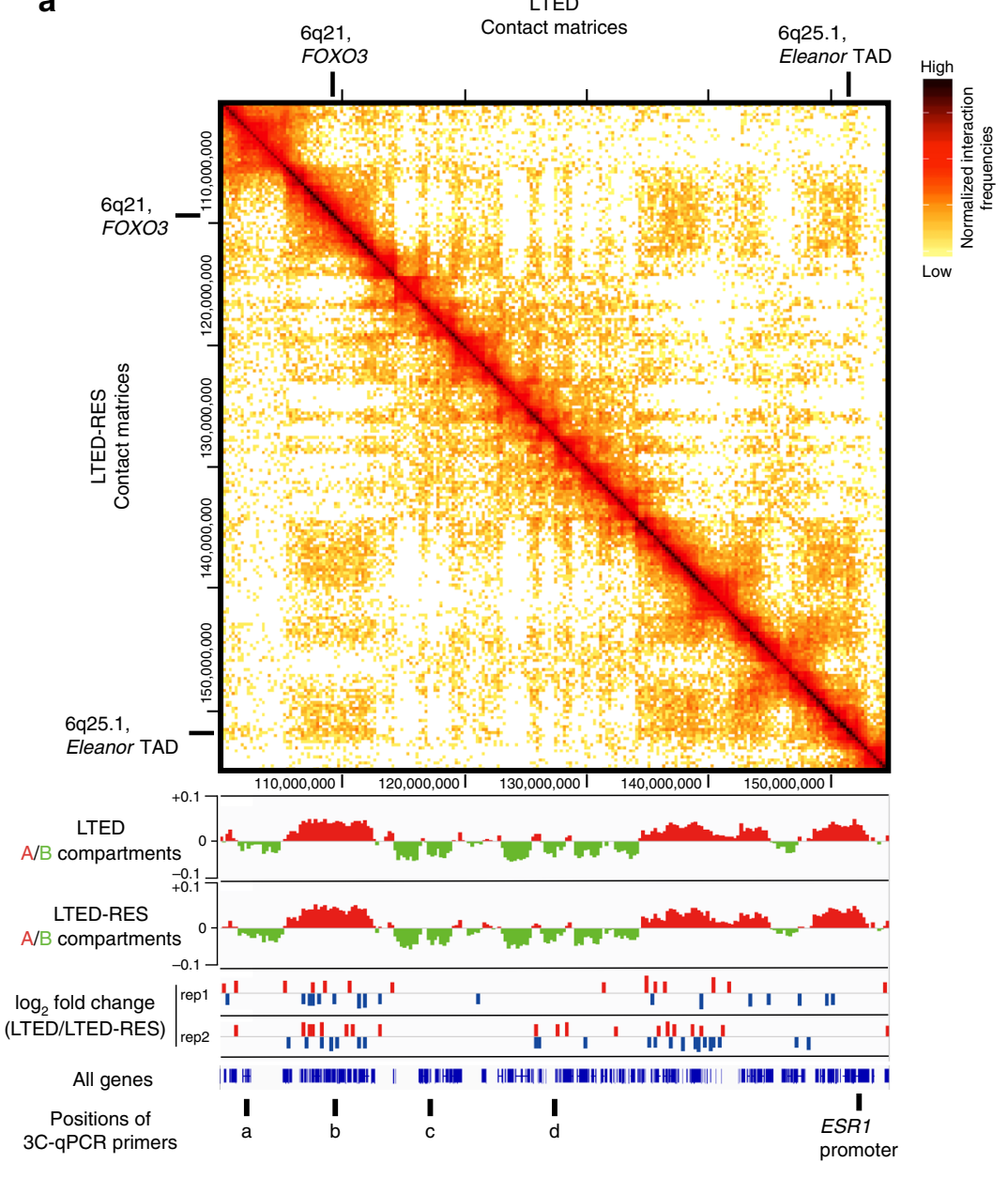

b

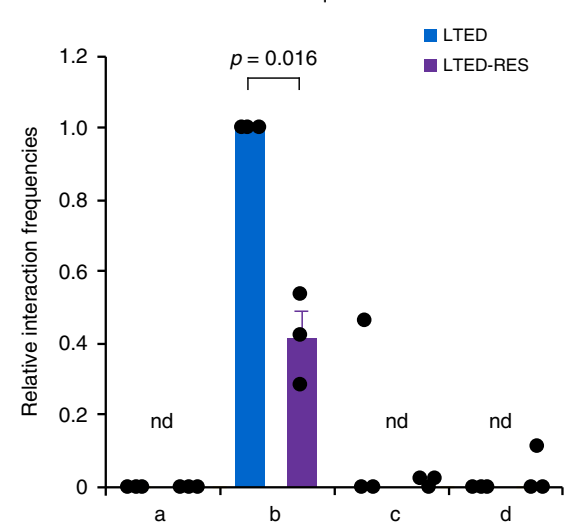

Fig. 3 Resveratrol treatment reduced the interaction between ESR1 and FOXO3 but maintained the FOXO3 gene activity. a The resveratrol treatment affected the interaction between the Eleanor topologically associating domain and other A compartments. Top: Contact matrices from Hi-C, binned at $250-$ $\mathrm{kb}$ resolution. The normalized interaction frequencies of long-term estrogen deprivation (LTED) and LTED-RES cells are shown above and below the diagonal line, respectively. Middle: A/B compartments and fold changes in the contact frequencies. Positions of gained (red) and reduced (blue) contacts with the ESR1 promoter are shown. Bottom: Positions of all genes and 3C-qPCR (chromosome conformation capture-quantitative polymerase chain reaction) primers, as in Fig. 2a. b 3C-qPCR shows the relative interaction frequencies of the b site decreased by resveratrol treatment. c Relative expression levels of genes at 6 q21 in LTED and LTED-RES cells. The LTED expression level was set to 1. Data presented in $\mathbf{b}, \mathbf{c}$ are representative of three independent experiments (mean \pm s.e.m.). $P$ values were calculated using unpaired, two-tailed, Student's $t$ test $(\star \star \star P<0.001$, n.d. $=$ not detected in at least one experiment) 
encoded on chromosome 6, and all of them were located in the A compartment in LTED cells (Supplementary Table 3). The transcription of Eleanors and Eleanor TAD genes may be upregulated through interactions with the active FOXO3 gene, which is paradoxically required to resist cell death in the transition from MCF7 to LTED.

The ESR1 promoter is transcribed in the forward direction. As we used the ESR1 promoter as the bait for the 4C-Seq analysis, we next investigated transcriptional condition in the ESR1 promoter that interacts with other active chromosomal regions. A majority of the transcriptional cis-elements are transcribed, including promoter/enhancer RNAs ${ }^{18,35}$. To search for TSS- or promoterassociated RNA (paRNA) in the Eleanor TAD, we aligned RNASeq and mRNA-Seq in which total RNA and poly $(A)^{+}$were used as templates, respectively. We found that the region proximal to the TSS of ESR1 (chr6: 152,128,354-152,128,780) was highly transcribed in the forward orientation in LTED cells (pa-Eleanor $(S)$; $S$ means sense strand), whereas it was moderately transcribed in the reverse direction in MCF7 cells (pa-Eleanor(AS); AS means antisense strand) (Fig. 4a, b). Both were suppressed by resveratrol treatment (Fig. 4a, b). The transcriptional switching from the reverse (in MCF7 cells) to the forward (in LTED cells) direction was characteristic of the ESR1 promoter region, because most (90\%) of the ESR1 gene region (chr6:152,128,814-152,424,408) was transcribed in the sense direction. The expression level of $p a-$ Eleanor in LTED cells was approximately seven times higher than that in MCF7 and HCC1428 cells (Fig. 4c and Supplementary Fig. 7a). qRT-PCR analysis confirmed that the expression of $p a-$ Eleanor was induced in LTED cells and was repressed by resveratrol treatment (LTED-RES) (Fig. 4c and Supplementary Fig. 7a). These observations are consistent with the fact that RNA polymerase II is more highly enriched at the promoter region in LTED cells than in MCF7 cells ${ }^{27}$. Our RT-PCR analysis using a series of primers showed that pa-Eleanor $(S)$ is a long ncRNA of at least $400 \mathrm{nt}$ in length and is not contiguous to exon 1 of ESR1 mRNA (Fig. 4d).

Because ESR1 is reported to be regulated by ER and FOXO3 transcription factors in breast cancer cell lines ${ }^{36-38}$, pa-Eleanor(S) levels could be affected by inhibiting their expression. However, qRT-PCR analysis showed that inhibition of ER, either by knockdown of ESR 1 mRNA with RNAi or specific degradation of ER protein with a chemical compound ICI 182,780 (Supplementary Fig. $8 \mathrm{~b}, \mathrm{e}$ ), had no effect on pa-Eleanor(S) expression (Supplementary Fig. 7b, c). The knockdown of FOXO3 also had little effect on either the level of pa-Eleanor(S) (Supplementary Fig. 7d) or the expression of Eleanor TAD genes in LTED cells (Supplementary Fig. 7e).

These results suggested that the amount and direction of transcription at the ESR1 promoter is highly regulated during the breast cancer adaptation to estrogen deprivation, which may define the level of ESR1 mRNA.

pa-Eleanor(S) is required for the Eleanor TAD. To investigate the molecular function of pa-Eleanor(S), we knocked it down in LTED cells using Antisense LNA GapmeR (LNA) (Fig. 5a), which resulted in strong suppression of genes inside the Eleanor TAD (RMND1, C6orf211, CCDC170, and ESR1) without affecting genes outside the Eleanor TAD (MTHFD1L and MTRF1L) (Fig. 5b). Interestingly, our FISH analysis showed that knockdown of $p a-$ Eleanor(S) downregulated the Eleanor cloud including itself (450E24) as well as all of the Eleanors from the entire $700 \mathrm{~kb}$ region (Fig. 5c, d) via an unknown mechanism. These results suggested that pa-Eleanor $(S)$ is important for the expression of all Eleanor IncRNAs, the formation of the Eleanor cloud, and the activation of genes in the Eleanor TAD. These cellular effects were strong, even though the efficacy of the knockdown itself was modest, suggesting the critical regulation of pa-Eleanor(S) in the nucleus. Since the results of the pa-Eleanor(S) knockdown (Fig. 5a-d) mirrored the effect of resveratrol treatment (LTEDRES), as shown in Fig. 1c, d, pa-Eleanor(S) may be a resveratrol target in LTED cells. In fact, in LTED cells, pa-Eleanor(S) sharply decreased upon resveratrol treatment (Fig. 4c).

Previously, we identified another Eleanor ncRNA, u-Eleanor, which activated ESR1 mRNA transcription and Eleanor cloud formation $^{27}$. To understand the regulation mode of pa-Eleanor and $u$-Eleanor, we investigated the effects of each knockdown. The $u$-Eleanor knockdown did not change the expression of $p a$ Eleanor (Supplementary Fig. 7f). In contrast, the pa-Eleanor(S) knockdown suppressed the $u$-Eleanor expression (Supplementary Fig. 7g), suggesting that pa-Eleanor upregulates the ESR1 transcription through $u$-Eleanor or may directly affect the expression of the ESR1 mRNA (Supplementary Fig. 7h).

We wondered whether the effects of pa-Eleanor $(S)$ knockdown affected events downstream of the reduced ESR1 mRNA or ER protein level and also whether knockdown of any Eleanor TAD RNA product could confer the same effects. To investigate these, we first knocked down ESR1 mRNA in LTED cells and found that the transcriptional levels of genes in the Eleanor TAD, including pa-Eleanor(S) and the Eleanor cloud, did not change (Supplementary Fig. 8a-d). Therefore, Eleanors are specific ncRNAs that play a role in the activation of the chromatin domain. Consistent with this result, ICI 182,780 treatment did not change the expression levels of either Eleanor TAD genes or pa-Eleanor $(S)$ (Supplementary Figs. 7c and 8e, f). Our previous reports also showed that the Eleanor cloud did not disappear after ICI 182,780 treatment ${ }^{27}$. Taken together, these results indicated that activation of the Eleanor TAD is not mediated by ER protein; rather, it depends on Eleanors.

Since Eleanors are important for LTED cell proliferation ${ }^{27}$, we wondered whether inhibition of pa-Eleanor(S) influenced cell growth. We found that pa-Eleanor(S) knockdown suppressed cell proliferation in both a time- and dose-dependent manner (Fig. $5 \mathrm{e}$ and Supplementary Fig. 8g). The results were similar to those obtained from resveratrol treatment of LTED cells, again suggesting that pa-Eleanor(S) is the target of resveratrol. LNA pa-Eleanor(S) specifically targets the forward-oriented transcript, which is predominant in LTED cells. Therefore, cell proliferation was inhibited more strongly in LTED than in MCF7 cells (Fig. 5e and Supplementary Fig. 8g). As the LTED cell formation recapitulates the development of endocrine therapy resistance, LNA for pa-Eleanor $(S)$ may be a promising therapeutic agent.

pa-Eleanor(S) mediates the long-range chromatin interaction. To investigate the function of pa-Eleanor $(S)$ in gene regulation through chromatin structures, we performed 3C-qPCR analysis. We found that the interaction between the ESR1 promoter and the b site at $6 \mathrm{q} 21$ reduced with pa-Eleanor $(S)$ knockdown in LTED cells (Fig. 6a), supporting the idea that ncRNA was involved in the long-range chromatin interaction. In contrast, transcriptional levels of the genes at 6q21 showed little-to-no change in the LTED cells with pa-Eleanor(S) knockdown (Fig. 6b). Knockdown of ESR1 mRNA or ER protein degradation with ICI 182,780 also did not cause any significant changes in gene expression in the 6q21 domain (Supplementary Fig. 9a, b). These results indicated that the ER transcription factor contributes very little to the ESR1 and FOXO3 genes in LTED cells. More importantly, activation of the FOXO3 gene in the $6 \mathrm{q} 21$ domain is autonomous and not dependent on a long-range chromosomal interaction via Eleanors, whereas transcriptional 
a
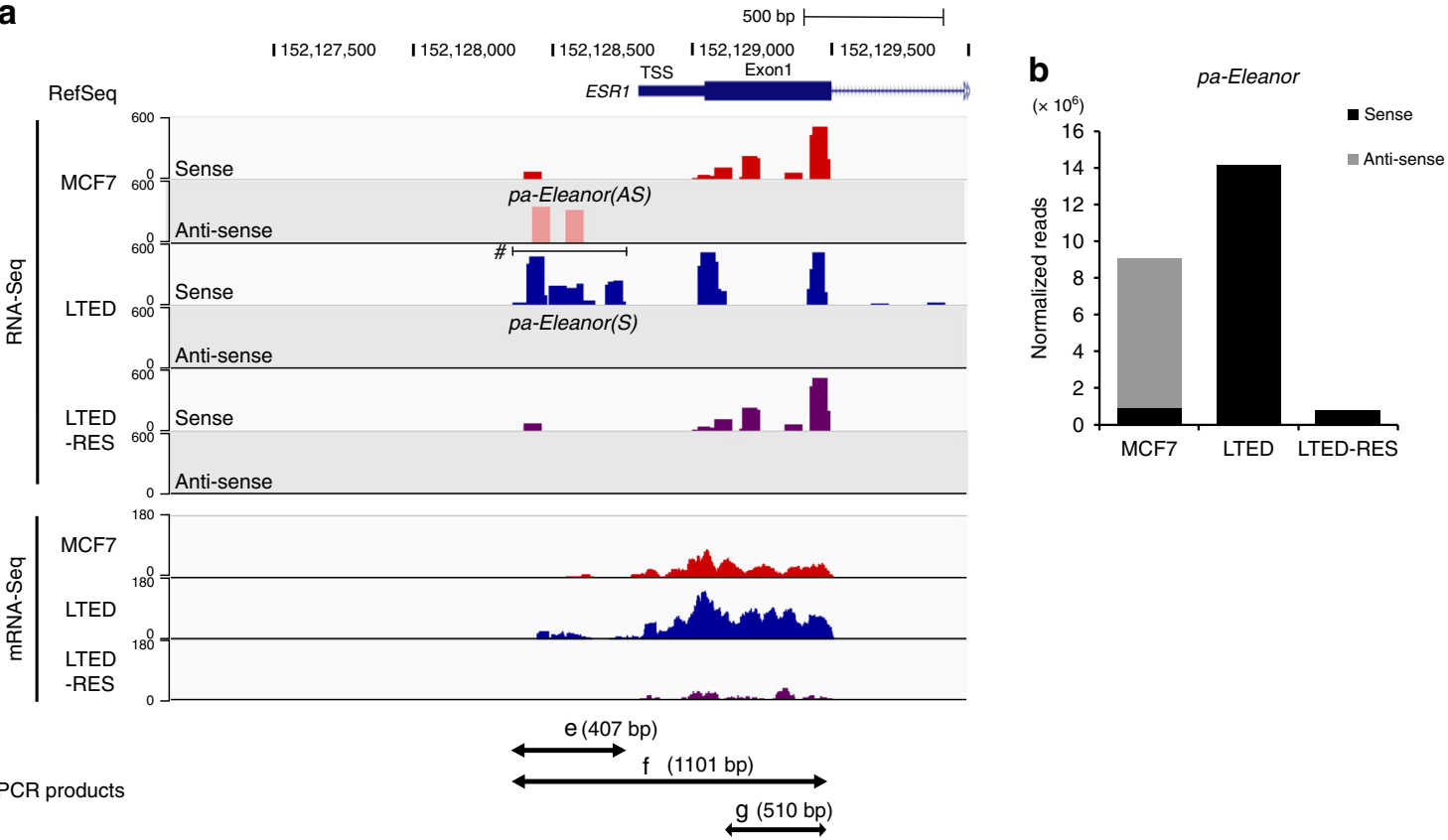

C
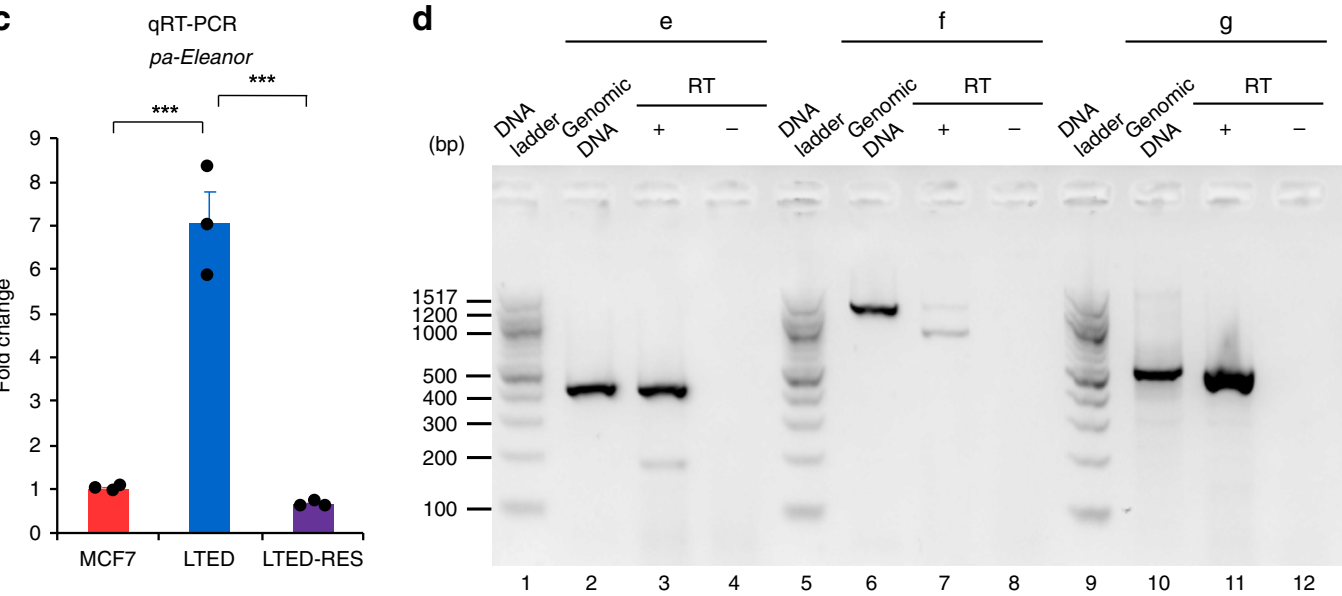

Fig. 4 The ESR1 promoter is transcribed differentially in MCF7 and long-term estrogen deprivation (LTED) cells. a Alignment of RNA-Seq and mRNA-Seq of the human ESR1 promoter-proximal region in the indicated cells ${ }^{27}$. Peaks in RNA-Seq indicate transcripts with sense (dark colors) and antisense (pale colors) orientation relative to the ESR1 gene. In MCF7 cells, antisense transcription was detected at the transcription start site (TSS), which then switched to opposite direction to produce pa-Eleanor(S) (denoted by the horizontal bar with hash (\#)) in LTED cells. Positions of the PCR products (e-g) in d are shown at the bottom. b Number of sequencing reads mapped at the TSS of the ESR1 gene. Numbers corresponding to sense and antisense RNA were counted and normalized according to the total number of mapped reads. $\mathbf{c}$ Expression levels of pa-Eleanor in the indicated cells determined with quantitative reverse transcription polymerase chain reaction ( $R$ RT-PCR). Values in MCF7 cells were set to 1. Data are representative of three independent experiments (mean \pm s.e.m.). $P$ values were calculated using unpaired, two-tailed, Student's $t$ test $\left({ }^{\star \star} P<0.001\right)$. $\mathbf{d}$ Local expression of $p a-E l e a n o r(S)$ at site e in LTED cells. RT-PCR to amplify predicted transcripts as shown in a (e-g) resulted in successful detection of the e-fragment (pa-Eleanor, lane 3 ) and g-fragment (exon1 of ESR1, lane 11) by electrophoresis but not the f-fragment (lane 7). These results indicate that pa-Eleanor(S) is not contiguous with the ESR1 mRNA. All primers and amplification conditions were validated with PCR in parallel with genomic DNA as a template and RT-PCR with and without reverse transcription (RT+ and -)

activities in the Eleanor TAD are dependent on this interaction. Consistent with this high level of FOXO3 transcription, our Annexin V staining and VB48 assays showed that LTED cells underwent apoptosis upon knockdown of pa-Eleanor(S) (Fig. 6c and Supplementary Fig. 10a-c). These results revealed a balanced chromosomal interaction between genes for cell proliferation (ESR1) and death (FOXO3) in LTED cells, mediated by Eleanors. Our data elucidated an ncRNA-mediated mechanism of chromatin interaction that underlies the characteristics of breast cancer cells primed for death.

\section{Discussion}

In this study, we found that pa-Eleanor(S) activates the genes in the Eleanor TAD and the Eleanor cloud formation in LETD cells (Fig. 7a). ESR1 and FOXO3, the genes for proliferation and apoptosis, respectively, are in close proximity and were paradoxically co-upregulated in LTED cells via Eleanors (Fig. 7b, left). Upon the inhibition of pa-Eleanor(S) by either knockdown or resveratrol treatment, Eleanors disappeared and the genes in the Eleanor TAD were repressed, whereas the FOXO3 activity was maintained and induced cell death (Fig. $7 \mathrm{~b}$, right). These findings 
a

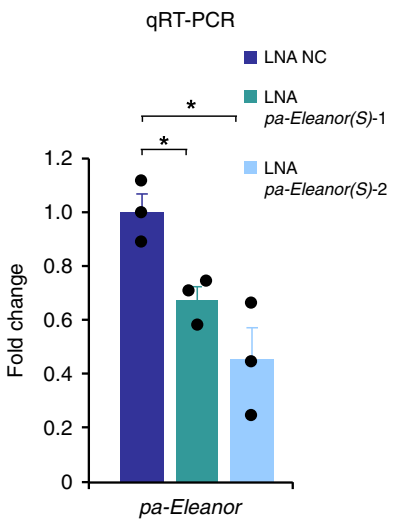

b

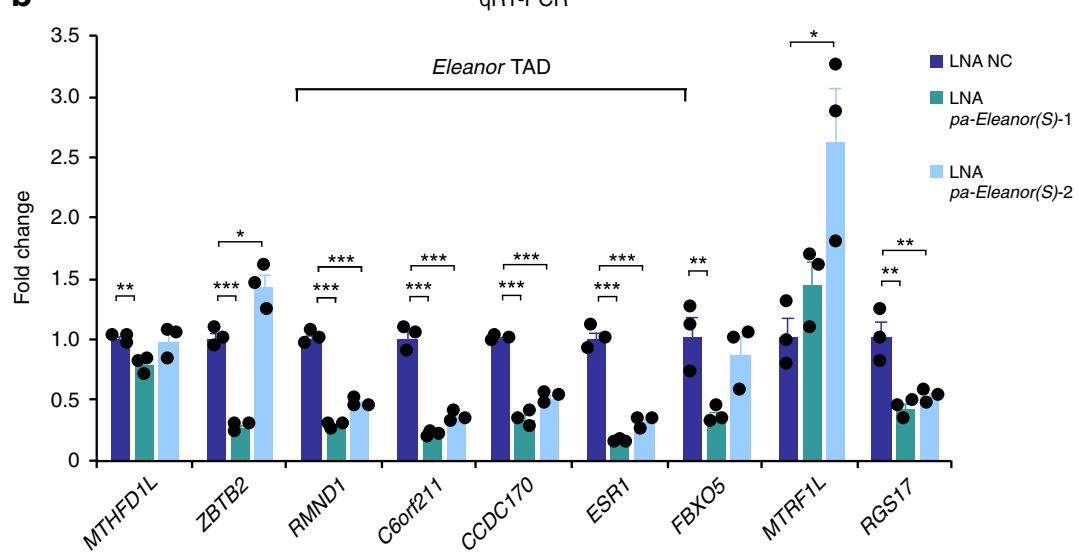

C
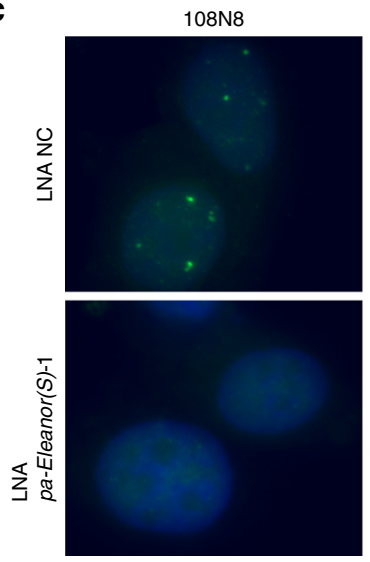

d $\left(\times 10^{4}\right)$

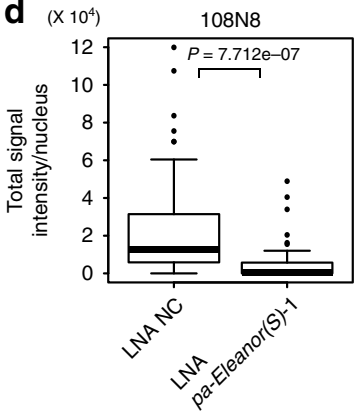

e

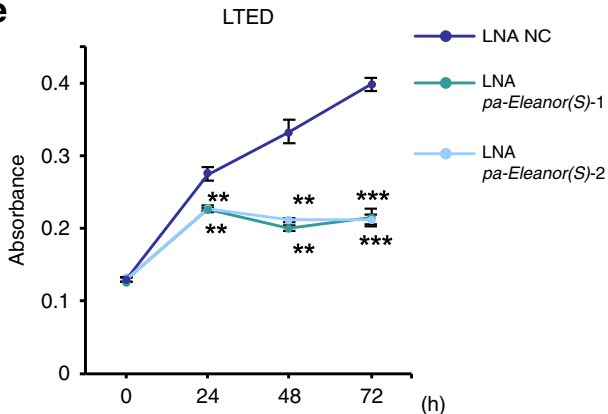

404G5
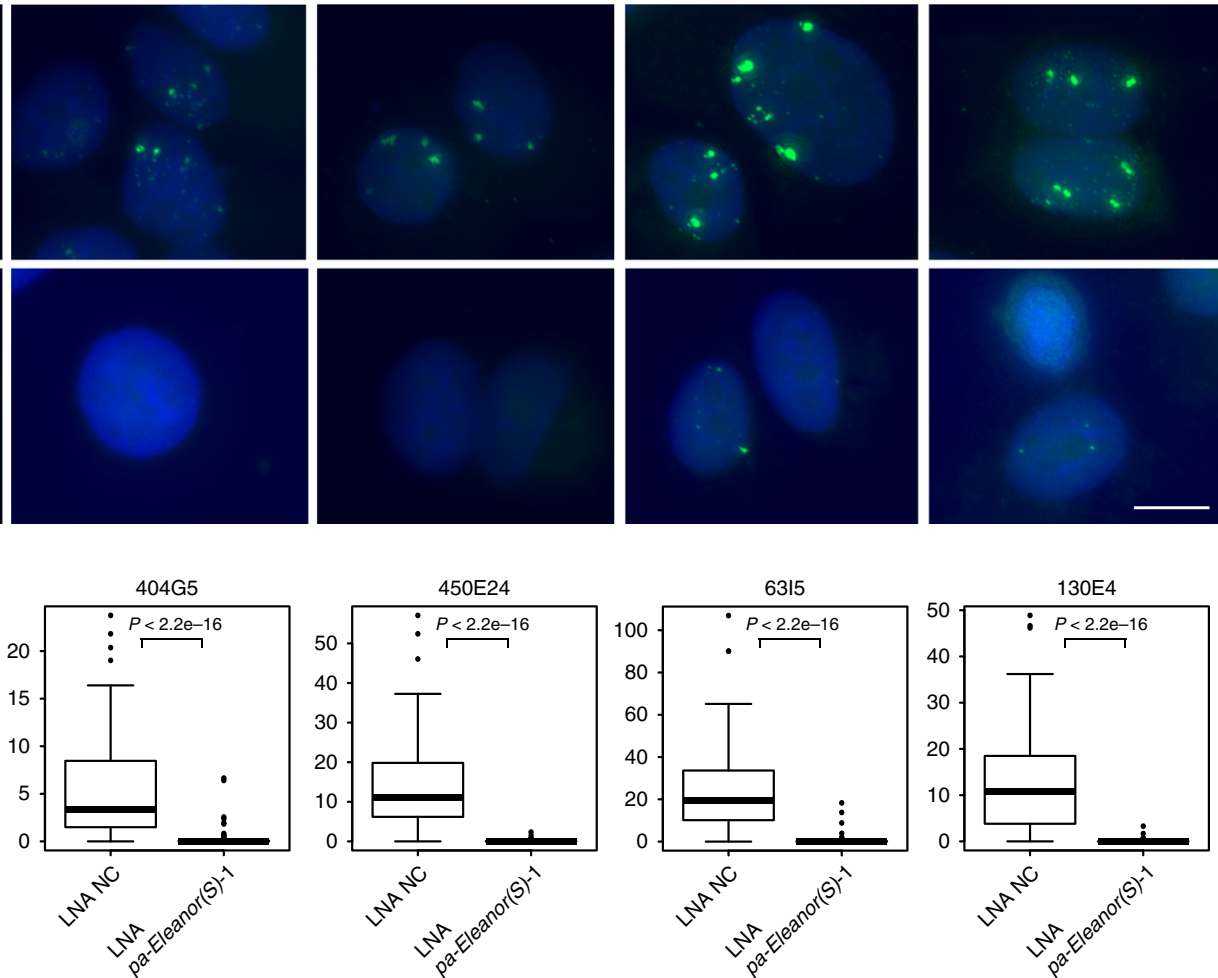

$130 \mathrm{E} 4$
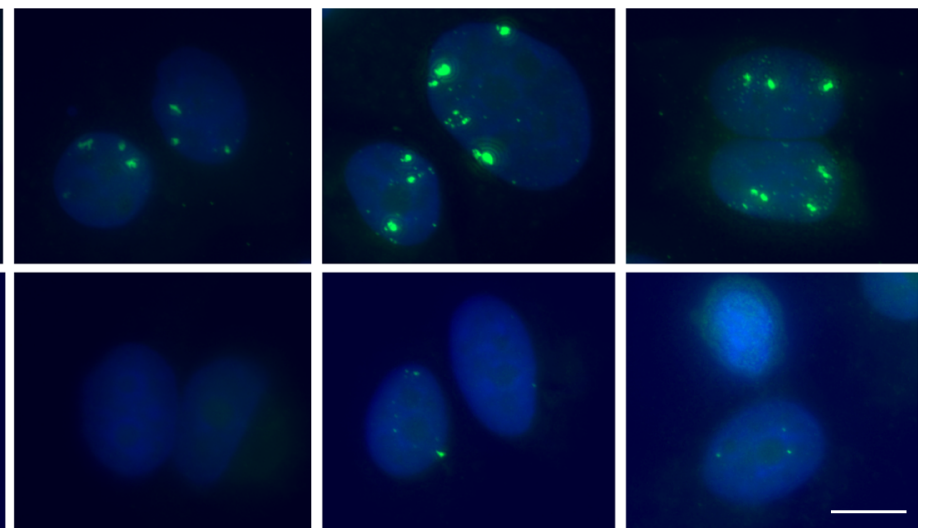

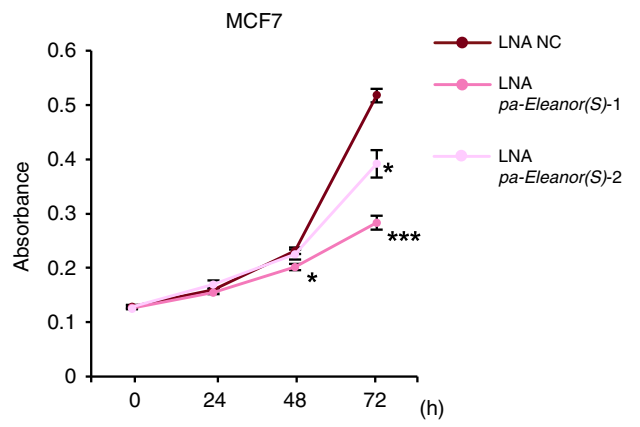

may represent the molecular mechanism underlying the apoptosisprone nature of endocrine therapy-resistant breast cancer cells.

We propose that the primary function of Eleanors is the activation of the ESR1 gene and neighboring genes in the Eleanor TAD (Fig. 7a). The long-range chromatin interaction between ESR1 and FOXO3 may also be mediated directly by Eleanors. Alternatively, the interaction may be the consequence of the activation of the Eleanor TAD. Upon Eleanor inhibition, ESR1 is inactivated and the interaction with FOXO3 is destabilized, but both genes are in the A compartment. The genes in the Eleanor TAD were repressed while they remained in the A compartment (Fig. 3a). This local chromatin re-wiring makes it possible to maintain the activity of FOXO3, while ESR1 is inactivated. Our findings provided an insight into the regulation of a chromatin 
Fig. 5 pa-Eleanor(S) plays a role in Eleanor topologically associating domain (TAD) formation and long-term estrogen deprivation (LTED) cell proliferation. a Quantitative reverse transcription polymerase chain reaction showed that pa-Eleanor(S) was knocked down with Antisense LNA GapmeR (LNA) in LTED cells. $\mathbf{b}$ Expression levels of genes in Eleanor TAD decreased upon pa-Eleanor(S) knockdown in LTED cells. Values of LNA for the negative control (LNA NC) were set to 1. c RNA fluorescence in situ hybridization (FISH) scanning analysis along the Eleanor TAD, with pa-Eleanor(S) knockdown in LTED cells. BAC clones used as FISH probes are indicated on the panels. The genomic positions covered by the BAC clones are shown in Fig. 1b (green bars). Nuclear Eleanor RNA foci were diminished with pa-Eleanor(S) knockdown. Scale bar, $10 \mu \mathrm{m}$. d Quantitative analysis of RNA FISH in c. Total signal intensities per nucleus in LTED cells were measured ( $n>40$ nuclei per sample). Boxplots shown are center line, median; box limits, upper and lower quartiles; whiskers, $1.5 \times$ interquartile range; points, outliers. $P$ values were calculated using two-tailed, Mann-Whitney $U$ test. e Inhibition of LTED cell proliferation due to paEleanor(S) knockdown. Time course analysis was performed after treatment of LTED (left) and MCF7 (right) cells with LNA. Data presented in $\mathbf{a}, \mathbf{b}$, e are representative of three independent experiments (mean \pm s.e.m.). $P$ values were calculated using unpaired, two-tailed, Student's $t$ test ${ }^{\star} P<0.05,{ }^{\star \star} P<$ $\left.0.01,{ }^{\star \star \star} P<0.001\right)$
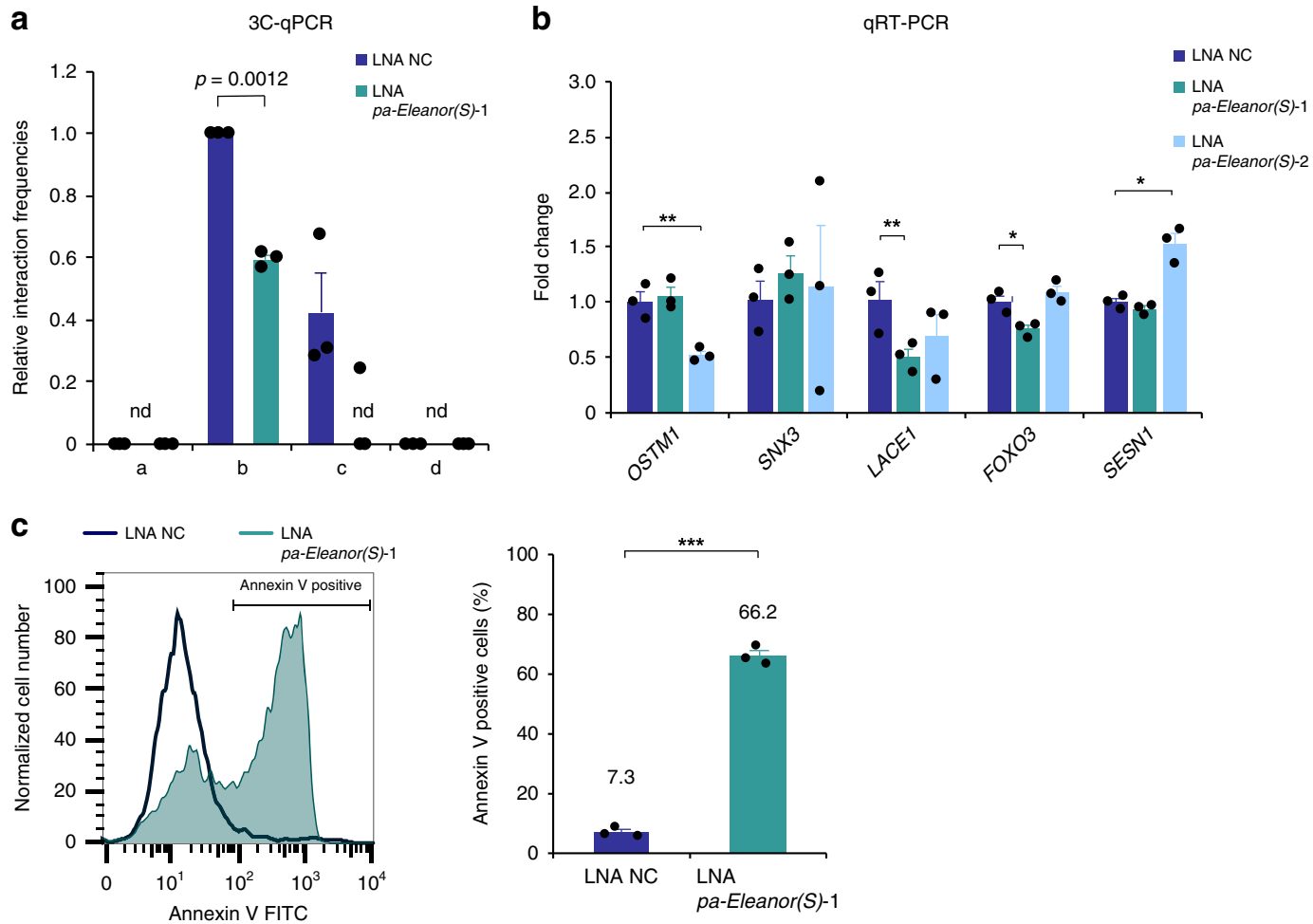

Fig. 6 Knockdown of pa-Eleanor(S) suppressed the long-range chromatin interaction without reducing FOXO3 gene activity and induced apoptosis in longterm estrogen deprivation (LTED) cells. a 3C-qPCR (chromosome conformation capture-quantitative polymerase chain reaction) analysis shows that paEleanor(S) knockdown reduced interaction between ESR1 and b sites at 6q21 in LTED cells. Interaction frequencies were measured between the ESR1 promoter and indicated positions (a-d; thick blue bars in Fig. 2a). b Expression levels of genes around the Eleanor topologically associating domain with paEleanor(S) knockdown in LTED cells. Quantitative reverse transcription polymerase chain reaction values of cells treated with Antisense LNA GapmeR for the negative control were set to 1. c Fluorescence-activated cell sorting (FACS) analysis of Annexin V-stained cells shows that knockdown of pa-Eleanor(S) increased apoptosis in LTED cells. A representative FACS profile is on the left. Quantification of the FACS analysis is on the right. Data presented in a-c are representative of three independent experiments (mean \pm s.e.m.). $P$ values were calculated using unpaired, two-tailed, Student's $t$ test $\left({ }^{\star} P<0.05\right.$,

${ }^{\star \star} P<0.01,{ }^{\star \star \star} P<0.001$, n.d. $=$ not detected in at least one experiment)

domain that underlies cancer fragility, in which the genes for cell growth and death interact via paRNA, and suggested that $p a-$ Eleanor(S) can be a potential therapeutic target for endocrine therapy-resistant breast cancer cells.

We found that Eleanor TAD was in the A compartment and also interacted with other A compartment TADs (Fig. 2). This is consistent with previous reports that describe how compartments of the same type are clustered in the nucleus ${ }^{12,39}$. Mechanisms for TAD formation involving chromatin structural proteins such as CTCF, cohesin, and the cohesin-loading factor Nipbl have been suggested, and local loop extrusion of a chromosome via these factors may be important ${ }^{16,40-43}$. According to publicly available data $^{44}$, these factors bind at the boundary of the Eleanor TAD, but they are not particularly enriched relative to regions within the TAD. Rather, we found that transcription of a cluster of ncRNAs (Eleanors) delineated the TAD in the A compartment in LTED cells. Other studies have suggested involvement of ncRNAs in $\mathrm{TAD}$ regulation ${ }^{13,42}$; however, the question of how general they are remains to be investigated. Because inhibition of Eleanors either by resveratrol treatment or specific knockdown decreased transcription of the genes inside the Eleanor TAD (Figs. 1c and $5 \mathrm{a}, \mathrm{b})$, the RNA products and RNA cloud formation may be critically important for active chromatin domain formation, although other factors including the ncRNA transcription process itself or DNA-RNA hybrid (R-loop) formation may also be important contributing factors. 


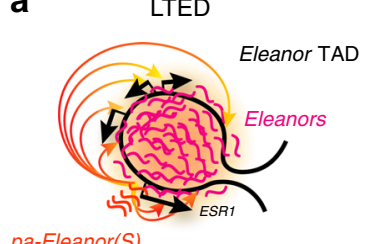

b

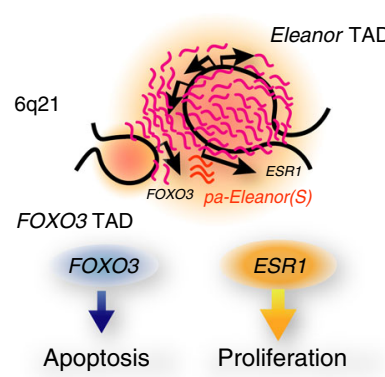

Eleanor inhibition

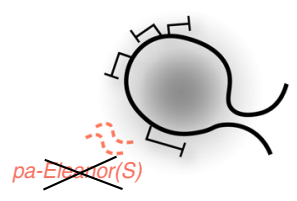

Eleanor inhibition

Unstable interaction
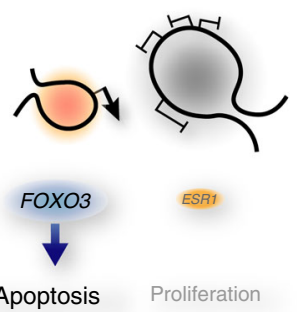

Fig. 7 Proposed model of Eleanor functions in long-term estrogen deprivation (LTED) cells. Eleanors play roles in activating genes within the Eleanor topologically associating domain (TAD) (a) and in mediating the long-range chromatin interaction (b). a In LTED cells, pa-Eleanor(S) activates the transcription of Eleanors and RNA cloud formation, thus activating the genes in the Eleanor TAD, including ESR1 (left). Eleanor inhibition represses the Eleanor TAD (right). $\mathbf{b}$ In LTED cells, the gene for proliferation (ESR1) and the gene for apoptosis (FOXO3) are in close proximity, as both are active in the A compartment. Upon inhibition of Eleanors by resveratrol or pa-Eleanor(S) knockdown, the long-range chromatin interaction decreased and the ESR1 gene was repressed, but the FOXO3 gene activity was maintained at a high level. These suggest a mechanism underlying the apoptosis-prone nature of LTED cells

Promoters are the key regulatory elements in long-range chromatin interactions ${ }^{45}$. In this study, we found that the ESR1 promoter interacted with $\mathrm{FOXO} 3$, a gene approximately $42.9 \mathrm{Mb}$ away from ESR1. We also found that the ESR1 promoter is bidirectionally transcribed in MCF7 cells, which is similar to other promoters exhibiting divergent transcription ${ }^{18-20}$. As ESR1 transcription was upregulated, the sense-stranded transcript (pa-Eleanor $(S))$ became more prevalent, which may be characteristic of active promoters ${ }^{18}$. Although the mechanism for the transcriptional switching is unknown, it is possible that RNA polymerase II transcription in the opposite direction may interfere with the movement of RNA polymerase II toward the ESR1 gene in MCF7 cells, which could be relieved in LTED cells. This idea is shared with transcriptional interference in yeast and other eukaryotes ${ }^{46,47}$.

It is clear that a long-range chromatin interaction between ESR1 and FOXO3 was enhanced by increased levels of pa-Eleanor (S) (Figs. 2d and 4c) and suppressed by inhibition of pa-Eleanor (S) (Figs. 3b, 4c, 5a, and 6a). This is the first demonstration of paRNA being responsible for a long-range chromatin interaction and transcriptional activation of genes in the TAD, probably through RNA cloud formation. An intriguing question is whether pa-Eleanor(S) shares molecular features with other ncRNAs involved in inter-chromosomal interactions, including HOTAIR and Firre ${ }^{48,49}$

The present study showed that inhibition of pa-Eleanor(S) suppressed ESR1 and the neighboring genes but did not affect the active gene FOXO3 (Figs. 5a, b and 6b), which promoted apoptosis in LTED cells. These findings suggested that activation of ESR1 is dependent on chromosomal interaction with the active FOXO3 gene via pa-Eleanor(S) and the Eleanor cloud in LTED cells. Although the possibility of the mutual activation of the two genes through the chromatin interaction cannot be excluded (Fig. 7b), it is more likely that FOXO3 is autonomously upregulated and provides the site of active transcription for ESR1. Therefore, disconnection of the physical interaction by Eleanor inhibition only affected genes in the Eleanor TAD, but not FOXO3, and could induce apoptosis (Figs. $5 \mathrm{a}, \mathrm{b}$ and $6 \mathrm{~b}, \mathrm{c}$ ). Because targeting of paRNA with antisense oligonucleotides, including LNA, is sequence-specific, it may be a good therapeutic strategy in comparison to targeting other epigenetic factors including histone modifications and their reader proteins that are distributed throughout the genome. Importantly, targeting of paRNA is also strand-specific, giving it an advantage over genome editing, which is not strand-specific.

ER and FOXO3 are intimately and paradoxically linked in breast cancer. They are co-upregulated in breast cancer tissues ${ }^{33,50}$ but have opposite biological effects. Estrogen induces transcriptional activity of ER while inactivating $\mathrm{FOXO}^{51}$. ER promotes estrogen-dependent breast cancer cell proliferation and tumorigenesis, whereas FOXO3 suppresses these effects ${ }^{38}$. Our apoptosis analysis showed that FOXO3 is responsible for apoptosis under estrogen deprivation (Supplementary Fig. 4c, d). Another study showed that the antidiabetic drug metformin induces apoptosis by FOXO3 upregulation in MCF7 cells ${ }^{52}$. FOXO3 binds to and activates the ESR1 promoter to induce ER expression in breast cancer ${ }^{37,53,54}$. Furthermore, FOXO3 binds to ER protein in the nucleus and inhibits its transcription factor activity $^{38,55}$. The present study indicated another type of crosstalk in which ESR1 and FOXO3 interact and are co-upregulated in the nucleus of LTED cells, which serve as a model for endocrine therapy-resistant breast cancer.

Co-upregulation of genes with seemingly counteracting functions may indicate the robustness and fragility of cancer. Cancer cells survive under various environmental changes via the regulation of gene expression, which allows them to acquire appropriate phenotypes ${ }^{56}$. For LTED cells undergoing unfavorable environmental changes (e.g., estrogen deprivation), it may be inevitable that $\mathrm{FOXO} 3$ is upregulated so that cells become primed for apoptosis ${ }^{8,9,57}$, representing the fragility of cancer. In contrast, ESR1 is upregulated for survival by interacting with the transcriptionally active $\mathrm{FOXO} 3$ gene through Eleanors, which represents cancer robustness. In other words, the majority of the LTED cells undergo cell death, at least partly due to upregulation of the apoptotic gene FOXO3, as shown in Supplementary Fig. 6. Cells may survive if the proliferation gene ESR1 acquires the ability to interact with the active TAD, including $F O X O 3$, via induction of Eleanors. Therefore, an intricate balance exists in LTED cells between apoptosis and proliferation. Targeting chromatin interaction disrupted this sensitive balance and made the cells prone to apoptosis (Fig. 6).

Resveratrol induces apoptosis in LTED cells ${ }^{34}$. The present study showed that pa-Eleanor(S) is a target of resveratrol (Fig. 4c). Because resveratrol and estrogen are structurally related, the molecular mechanism presented in this report may explain the efficacy of estrogen additive therapy following AI treatment ${ }^{8,9,11}$. Our study reveals a chromatin interaction mechanism via ncRNAs and sheds light on cancer robustness and fragility.

\section{Methods}

Cell culture. MCF7 (ATCC) and HCC1428 (ATCC) cells were cultured in RPMI 1640 (Sigma) supplemented with 10\% fetal bovine serum (FBS; Corning). For LTED cells, MCF7 or HCC1428 cells were grown in phenol red-free RPMI 1640 (Wako) containing 4\% dextran-coated charcoal-stripped FBS (Thermo Fisher Science) for 3-8 months. LTED cells were treated with $100 \mu \mathrm{M}$ resveratrol (Sigma) for $24 \mathrm{~h}$ (LTED-RES cells) or with $100 \mathrm{nM}$ ICI 182,780 (Tocris) for $48 \mathrm{~h}$ (LTED-ICI cells). Cells were cultured at $37^{\circ} \mathrm{C}$ and $5 \% \mathrm{CO}_{2}$. During the course of this study, all cell lines were tested for mycoplasma contamination. 
4C template preparation. The experimental strategy for $4 \mathrm{C}$ analysis ${ }^{58}$ is shown in Supplementary Fig. 1. In brief, at least $10^{7}$ MCF7 or LTED cells were harvested and crosslinked with $1 \%$ formaldehyde for 5 min with rotation at room temperature, followed by quenching with a final concentration of $125 \mathrm{mM}$ glycine and centrifugation for $10 \mathrm{~min}$ at $400 \times g$ and $4^{\circ} \mathrm{C}$. Nuclei were digested overnight with the first restriction enzyme at $37^{\circ} \mathrm{C}$, followed by proximity ligation with T4DNA ligase (TaKaRa, 2011A) for $4 \mathrm{~h}$ at $16^{\circ} \mathrm{C}$ and then kept for $30 \mathrm{~min}$ at room temperature. Chromatin was de-crosslinked with $2 \%$ proteinase $\mathrm{K}$ in an overnight digestion at $65^{\circ} \mathrm{C}$. DNA was extracted with phenol-chloroform and precipitated with ethanol and then further digested with the second restriction enzyme overnight at $37^{\circ} \mathrm{C}$ and subsequently ligated in T4 DNA ligase. Resultant DNA circles were 4C templates, which were precipitated in ethanol with glycogen as a carrier $(20 \mu \mathrm{g} / \mathrm{mL})$. The interacting DNA segments captured were amplified with PCR using LA Taq (TaKaRa, RR002A). For 4C experiments, two independent sets (exp-A and exp-B) were performed using different restriction enzymes and primers. For exp-A, DpnII (NEB R0543M) was used as the first restriction enzyme and Csp6I (Thermo, ER0211) as the second restriction enzyme. Primers for 4C library construction for exp-A were as follows: DpnII forward: 5'-TCTGGAAAGACGTTCTTGATC-3'; Csp6I reverse: 5'-CTGGATGAATGGCAGTGG-3'. For exp-B, HindIII (TaKaRa, $1060 \mathrm{AH}$ ) was used as the first restriction enzyme and DpnII (NEB R0543M) as the second restriction enzyme. Primers for $4 \mathrm{C}$ library construction for exp-B were as follows: HindIII forward: 5'-AACATAACCTCAGGTCACGAAC-3'; DpnII reverse: 5'-GCACATAAGGCAGCACATTAG-3'. Each experimental set was repeated for two independent replicates.

4C-Seq and data analysis. The 4C-Seq library was prepared using a NEBNext Ultra DNA Library Prep Kit, according to the manufacturer's protocol, and sequenced using the Illumina HiSeq1500 system (Illumina, San Diego, CA, USA). The sequenced reads were mapped to the human reference genome (hg19) using the Bowtie 2 software (v2.1.0) with default parameters ${ }^{59}$. Uniquely mapped reads were selected. PCR duplicates were removed from mapped reads using SAMtools $(\mathrm{v} 0.1 .18)^{60}$. The mapped reads in every 500 -kb non-overlapping genomic segment (bin) were counted, and the counts were normalized as read per million (RPM) scores. The differences in RPM scores between $4 \mathrm{C}$ and input control data $\left(\mathrm{RPM}_{4 \mathrm{C}}\right.$ $-\mathrm{RPM}_{\text {input }}$ ) were calculated as $4 \mathrm{C}$ signal intensity of each bin. Furthermore, we analyzed our $4 \mathrm{C}$-Seq by using the $4 \mathrm{C}$ pipeline developed by Tanay and de Laat groups $^{61}$.

For Supplementary Fig. 2a, we performed analysis of variance followed by Tukey-Kramer test to calculate the adjusted $P$ values and determined the valley regions in the $4 \mathrm{C}$-Seq peaks. The calculation was done for 20 bins of each $100 \mathrm{~kb}$ along the region of chr6: 151,000,000-153,000,000. Prior to the calculation, the 4Cseq reads were normalized at every $10 \mathrm{~kb}$.

Hi-C experiment. Hi-C experiments ${ }^{62}$ using LTED and LTED-RES cells were performed as follows. Briefly, cells were crosslinked with $1 \%$ formaldehyde for $10 \mathrm{~min}$ at room temperature, followed by quenching with a final concentration of $125 \mathrm{mM}$ glycine. In total, $1-2 \times 10^{6}$ fixed cells were used for the following steps. Nuclei were extracted and digested overnight with $200 \mathrm{U}$ DpnII (NEB, R0543S) at $37^{\circ} \mathrm{C}$ while shaking (Eppendorf ThermoMixer ${ }^{\oplus} \mathrm{C}$ ). Next, the nuclei were collected by centrifugation and washed with $1 \times$ NEB buffer 2 and then incubated in Fill-in Mix [including Biotin-14-dATP (Invitrogen, 19524-016), dGTP, dTTP, and dCTP with $15 \mathrm{U}$ of Klenow (NEB, M0210L), $1 \%$ Triton X-100)] for $1 \mathrm{~h}$ at $25^{\circ} \mathrm{C}$. The nuclei were then collected by centrifugation and incubated in Ligation Mix $[1 \times \mathrm{T} 4$ DNA ligase buffer (NEB, B0202S) with 1800 cohesive-end ligation units (CEU) of T4 DNA ligase (Invitrogen, 15224-025), $1 \%$ Triton X-100] for $6 \mathrm{~h}$ at $16^{\circ} \mathrm{C}$ while shaking. Samples were treated with RNase A and were de-crosslinked with proteinase $\mathrm{K}$ overnight at $65^{\circ} \mathrm{C}$. DNA was extracted with phenol-chloroform and precipitated with ethanol. Purified DNA samples were checked for quantity and quality by the Qubit dsDNA HS Assay Kits (Thermo Fisher Scientific) and TapeStation 4200 (Agilent), respectively.

For next-generation sequencing libraries, Hi-C DNAs were mixed with T4 DNA pol mix [including dATP, dGTP, and $3 \mathrm{U}$ of T4 DNA polymerase (NEB, M0203S)] and incubated for $30 \mathrm{~min}$ at $37^{\circ} \mathrm{C}$. These DNAs were sonicated (150-400 bp) using the DNA Shearing System S220 (Covaris) and size-selected using AMPure XP beads (Beckman Coulter). The size-selected DNAs were captured with Dynabeads ${ }^{\oplus}$ M-280 Streptavidin (Thermo Fisher Scientific). For the sequencing library preparations, a KAPA LTP Library Preparation Kit (KAPA Biosystems) was used, and the purification was performed with AMPure XP beads. The quality of the libraries was monitored with TapeStation 4200, and paired-end sequencing (150-bp read length) was performed using Hiseq X Ten (Illumina). We obtained $\sim 70$ million paired-end reads per sample.

Hi-C analysis. For the LTED and LTED-RES Hi-C data, the paired-end reads were individually mapped to the human genome (hg19) using the Juicer (v1.5) pipeline ${ }^{63}$. We used mapped reads with MAPQ $>30$ to generate.hic files for further analyses. For generating $\mathrm{A} / \mathrm{B}$ compartment profiles at $250-\mathrm{kb}$ resolution, the first eigenvector of the Pearson's correlation matrix was used ${ }^{64}$. For the analysis of the differences in the $\mathrm{Hi}-\mathrm{C}$ contact frequency, we extracted the number of interactions (i.e., reads) revealed by $\mathrm{Hi}-\mathrm{C}$ for every $250-\mathrm{kb}$ bin using the Juicer tools ${ }^{63}$ from the hic file, and the differences in the interaction frequencies on chr6 (chr6:100,000,000-155,000,000) were calculated using the R package HiCcompare $^{65}$. The normalized interaction frequencies and fold-change differences were $\log$ transformed and displayed as a heat map, using the R package ggplot2. Changes in interaction frequencies with the Eleanor TAD were computed, and significant changes were extracted using HiCcompare.

According to the method developed by the Yue and colleagues ${ }^{66}$, we created the Binary Upper Triangular Matrix (BUTLR) File Format, using the published normalized Hi-C matrix data of MCF7 at 40-kb resolution (GEO accession: GSE66733, Supplementary file $)^{29}$ and visualized it as a contact matrix on a 3D Genome Browser (http://promoter.bx.psu.edu/hi-c/view.php). The A/B compartment profiles at $250-\mathrm{kb}$ resolution and the positions of the TAD boundaries were obtained ${ }^{29}$.

The contact matrices binned at $10-40-\mathrm{kb}$ resolutions with the positions of the TADs for the IMR90, HMEC, GM12878 (GEO accession: GSE63525) ${ }^{16}$, and T47D cells (GEO accession: GSM2827515) ${ }^{44}$ were obtained from the 3D Genome Browser ${ }^{66}$

Analysis of overlap between $\mathbf{4 C}$ peaks and genome regions. We created $4 \mathrm{C}$-Seq bed files at $250-\mathrm{kb}$ resolution $\left(\mathrm{RPM}_{4 \mathrm{C}}-\mathrm{RPM}_{\text {input }}\right.$ above the defined threshold) using deepTools (v3.13) ${ }^{67}$ and bedtools (v2.27.1) ${ }^{68}$. The files for A/B compartment peaks and those for genes upregulated or not upregulated in LTED cells were compared with the 4C-Seq files, using the mergePeaks tool of Homer (v4.10) ${ }^{69}$

Chromatin immunoprecipitation (ChIP)-qPCR. ChIP assays were performed according to the Upstate Biotechnology protocol ${ }^{70}$ with modifications. Cells were crosslinked with $1 \%$ formaldehyde for $5 \mathrm{~min}$ at room temperature. Twenty $\mu \mathrm{L}$ of Dynabeads M-280 Sheep Anti-Mouse IgG (Thermo Fisher Scientific) were bound with $2 \mu \mathrm{g}$ of a Phospho RNA Polymerase II CTD antibody (Ser5) (MABI0603). Nuclei were isolated and sonicated with a Bioruptor (20 times, $30 \mathrm{~s}$ ON/30 s OFF, output; high) (COSMO BIO) to generate DNA fragments. The DNA fragments were incubated overnight at $4^{\circ} \mathrm{C}$ with magnetic beads bound with the antibodies. The beads were washed and de-crosslinked for $4 \mathrm{~h}$ at $65^{\circ} \mathrm{C}$. RNaseA and Proteinase $\mathrm{K}$ were used for RNA and protein digestion, respectively, and the DNA was purified with a QIAquick PCR Purification Kit (Qiagen). DNA enrichment was determined by a qPCR analysis with a Step One Plus system (Applied Biosystems), using SYBR green fluorescence. The input DNA was used to make a standard curve to determine the DNA enrichment. Primer sequences are listed in Supplementary Table 7.

3C quantitative PCR. At least $10^{7}$ MCF7 and LTED cells were crosslinked using $1 \%$ formaldehyde in medium for $5 \mathrm{~min}$ at room temperature with rotation. Cells were lysed using NP- 40 lysis buffer for $15 \mathrm{~min}$ at $4{ }^{\circ} \mathrm{C}$. Nuclei were washed and resuspended in restriction buffer and then digested with $1 \mathrm{U} / \mu \mathrm{L}$ HindIII $(\mathrm{TaKaRa}$, $1060 \mathrm{AH})$ overnight at $37^{\circ} \mathrm{C}$ while shaking. After inactivating the restriction enzymes by heating at $65^{\circ} \mathrm{C}$ for $20 \mathrm{~min}$ in the presence of $1.6 \%$ sodium dodecyl sulfate (SDS), proximity ligation was done in $7 \mathrm{~mL}$ total volume using $350 \mathrm{U}$ T4DNA ligase (TaKaRa, 2011A) for $4 \mathrm{~h}$ at $16^{\circ} \mathrm{C}$ and then kept for $30 \mathrm{~min}$ at room temperature. After de-crosslinking and RNase treatment, the DNA was purified by phenol-chloroform extraction and ethanol precipitation. The $3 \mathrm{C}$ template was cleaned using the wizard DNA cleanup system (Promega, A7280). The ligated products (3C template) were assessed using $\mathrm{qPCR}$ with the ABI Prism 7300 and Step One Plus system (Applied Biosystems) using TaqMan Fast advanced mastermix (ABI). Primers in this analysis are listed in Supplementary Table 6. For the standard qPCR curve, we used purified BAC DNAs that were digested and ligated. The BAC DNAs containing the target genomic loci were ESRIp-BAC for reference and another locus position for a-d (see "BAC clones" section for details).

Quantitative PCR of genomic DNA for copy number measurement. Genomic DNA from MCF7 and LTED cells was isolated using a Qiagen DNeasy Kit (Cat. No. 69504), according to the manufacturer's protocol. One column was used for cells grown in 10-cm dish. qPCR was performed with SYBR green fluorescence on an ABI Prism 7300 system (Applied Biosystems). The calibrator was prepared by mixing BAC clones covering tested regions in equimolar amounts (see "BAC clones" section). This mixture was serially diluted to produce a standard curve for normalization against amplification efficiency of each primer set used. Values were further normalized to the genomic DNA of a reference gene, GAPDH, before calculating relative fold changes. Primer sets used for analysis are listed in Supplementary Table 6.

PCR with reverse transcribed RNA. Total RNA was isolated from cultured cells with TRIzol (Invitrogen). RT was carried out with Rever Tra Ace qPCR RT Master Mix with gDNA Remover (TOYOBO). qPCR was performed with SYBR green fluorescence on an ABI Prism 7300, 7500, and Step One Plus system (Applied Biosystems). Values were normalized to GAPDH expression before calculating relative fold changes. Primer sets used for analysis are listed in Supplementary Table 4. For RT-PCR in Fig. 4d, PCR-amplified products were run on agarose gel and stained with SYBR Gold (Thermo Fisher Science). The genomic DNA and the cDNA with and without RT from the extracted RNA were amplified with PCR system on specified number of cycles (Applied Biosystems) using Q5 High-Fidelity DNA Polymerase (NEB). Genomic DNA was used as a control. Primer sets used for analysis are listed in Supplementary Table 8. 
RNA stability assay. LTED cells were treated with $1 \mu \mathrm{g} / \mathrm{mL}$ actinomycin D (Wako) for 15 min to stop RNA synthesis, and then the residual RNA levels were measured at every $60 \mathrm{~min}$ for $300 \mathrm{~min}$. RNA isolation, RT, and PCR analysis were performed as described above. $c M y c$ was used as a control for unstable RNA. The primer sets are listed in Supplementary Table 4

Immunoblotting. To prepare the total cell lysate, cells were dissolved in SDS sample buffer containing benzonase (Sigma). Proteins were separated by SDS-polyacrylamide gel electrophoresis and then transferred to a polyvinylidene difluoride membrane, Hybond-ECL (GE Healthcare). The membrane was blocked for $1 \mathrm{~h}$ with phosphate-buffered saline (PBS) containing 10\% nonfat dry milk and then incubated with primary antibodies against ERa (Santa Cruz Biotechnology, sc-543, 1:1000) and Actin (Sigma, A2103, 1:1000) in Can Get Signal immunostain Solution A (Nacalai Tesque), overnight at $4{ }^{\circ} \mathrm{C}$. The membrane was washed and incubated with secondary antibodies in Can Get Signal immunostain Solution B (Nacalai Tesque) for $60 \mathrm{~min}$ at RT. After the membrane was washed, the signals were visualized with Chemi-Lumi One Ultra solution (Nacalai Tesque) and detected by an Amersham Imager 600 (GE Healthcare). The uncropped and unprocessed scans of the blots with molecular marker (MagicMark ${ }^{\mathrm{TM}}$ XP, Sigma) are available in Supplementary Fig. 11.

BAC clones. BAC DNA used as FISH probes and 3C-qPCR that cover the Eleanor TAD (Chr6 q25.1), Chr6 q21 (for FISH), and sites a-d are listed in Supplementary Table 5. BAC DNA was prepared using a large-construct DNA Isolation Kit (Qiagen).

Fluorescence in situ hybridization. DNA FISH was performed as follows. Cells grown on coverslips were fixed with $4 \%$ formaldehyde and $0.5 \%$ Triton X-100 in PBS for $15 \mathrm{~min}$ and then permeabilized with $0.5 \%$ saponin and $0.5 \%$ Triton X-100 in PBS for $20 \mathrm{~min}$. Samples were immersed in $20 \%$ glycerol in PBS for $30 \mathrm{~min}$ and subjected to four cycles of freeze-thawing by freezing the cells in liquid nitrogen for $30 \mathrm{~s}$ each time and then thawing at room temperature. The cells were then treated with $0.1 \mathrm{~N} \mathrm{HCl}$ for $15 \mathrm{~min}$. For denaturation and hybridization, the cells were incubated in hybridization mixtures $(2 \times$ SSC, $50 \%$ formamide, $10 \%$ dextran sulfate, $1 \mathrm{mg} / \mathrm{mL}$ tRNA, and $5-10 \mu \mathrm{g} / \mathrm{mL}$ probe DNA) at $75^{\circ} \mathrm{C}$ for $4-10 \mathrm{~min}$ (for denaturation of genomic DNA) and then at $37^{\circ} \mathrm{C}$ for $48-72 \mathrm{~h}$ (for hybridization). For RNA FISH, the same protocol was used, except for the genomic DNA denaturation step. BAC probes were labeled with biotin or digoxigenin in a nick translation mixture (Roche), according to the manufacturer's protocol. After hybridization, the cells were washed with $2 \times$ SSC and $50 \%$ formamide at $37^{\circ} \mathrm{C}$ for $5 \mathrm{~min}$, followed by $2 \times \mathrm{SSC}$ at $37^{\circ} \mathrm{C}$ for $5 \mathrm{~min}$. FISH signals were detected with fluorescein isothiocyanate-anti-digoxigenin (Roche) or Cy3-streptavidin (Jackson ImmunoResearch). DNA was counterstained with 4,6-diamidino-2-phenylindole.

Microscopic and image analysis. FISH images were obtained using an IX-71 microscope (Olympus) equipped with a $60 \times$ NA1.0 Plan Apo objective lens, a cooled CCD camera (Hamamatsu), and image acquisition software (Lumina Vision Version 2.4; Mitani Corporation). For comparison of the effects of resveratrol, small interfering RNA (siRNA), and LNA treatments, identical image capture conditions were used within a set of experiments. Image stacks of $3 \mathrm{D}$ data sets were collected at $0.5-1.0-\mu \mathrm{m}$ intervals through the $z$ axis, subjected to projections. The signal intensity of RNA FISH was analyzed using a Cellomics CellInsight with HCS studio cell analysis software (Thermo Fisher Scientific).

FISH images in Fig. 2b were obtained with a confocal laser-scanning microscope (LSM 780, Carl Zeiss) equipped with $\times 63 / 1.4$ Plan-Apochrome objective lens and a cooled CCD camera (Carl Zeiss). Images were acquired using the LSM software (Carl Zeiss).

Transfection of cells with LNA and siRNA. Cells were transfected with Antisense LNA GapmeR Negative Control A, LNA pa-Eleanor(S)-1, LNA pa-Eleanor(S)-2 (TaKaRa), siRNA-GL3, siRNA-ESR1 (Nippon EGT), siRNA-FOXO3 (CST, \#6303), and siRNA-SNX3 (Santa Cruz Biotechnology, sc-41351) using RNAiMAX (Invitrogen). Target sequences for each Antisense LNA and siRNA are listed in Supplementary Table 9 . The cells were analyzed 48 or $72 \mathrm{~h}$ after transfection for qRTPCR, RNA FISH, and fluorescence-activated cell sorting (FACS) analyses.

Transfection of cells. Cells were transfected with HA-FOXO3a wild type (addgene \#1787) using Lipofectamine 2000 (Invitrogen). The cells were monitored $24 \mathrm{~h}$ after transfection by qRT-PCR and FACS analyses.

Cell proliferation assay. Cell proliferation assay was performed using the Cell Counting Kit-8 (CCK-8; Dojindo). Briefly, cells were seeded in 96-well plates; incubated at $37^{\circ} \mathrm{C}$ for $24 \mathrm{~h}$; and transfected with LNA, siRNA, or plasmid. After adding CCK- 8 and incubating for $1 \mathrm{~h}$ at $37^{\circ} \mathrm{C}$ in a humidified $\mathrm{CO}_{2}$ incubator, the degree of yellow color in the medium was measured at an absorbance of $490 \mathrm{~nm}$ by a microplate reader (BIO-RAD)
Apoptosis assay. Cells were stained using an Annexin-V-FLUOS Staining Kit (Roche), according to the manufacturer's protocol. For FACS analysis, a mixed solution of floating and attached cells were prepared, incubated for $15 \mathrm{~min}$ with Annexin V-FLUOS and propidium iodide (PI) labeled solution, and then analyzed with a FACS analyzer (BD FACSCanto ${ }^{\mathrm{Tm}}$ II and BD FACSCalibur ${ }^{\mathrm{m}}$ ). For further analysis, we used the FlowJo program (v10.4.1). For microscopic analysis of apoptotic cells, cells grown on a dish were washed with PBS, incubated for $15 \mathrm{~min}$ with Annexin V-FLUOS-labeled solution, and then observed under a microscope CKX53 (Olympus) with CellSens standard program (v 1.16). To count cells, merged images were created using ImageJ (v1.49) ${ }^{71}$.

Apoptosis-related gene analysis. Apoptosis-related genes (Qiagen, https://www. qiagen.com/jp/resources/resourcedetail?id=e5252c51-7513-44a0-b66d$927 \mathrm{c} 53 \mathrm{e} 0$ eeb2\&lang=en) were extracted from the RNA-Seq files ${ }^{27}$, and the expression levels were compared in MCF7 and LTED cells.

Cell vitality assay. Cell vitality was assessed using VitaBright-48 (VB48, Chemometec), which binds to reduced glutathione in the cell and emits light, using an Automated Cell Analyzer (NucleoCounter NC-250, Chemometec). A mixed solution of floating and attached cells was used for VB48 staining.

ChIP-Seq and chromatin interaction analysis by paired-end tag sequencing (ChIA-PET) data used in this study. ChIP-Seq and ChIA-PET data of MCF7 cells (Supplementary Table 10) were obtained from ENCODE Consortium through the UCSC genome browser (University of California, Santa Cruz) ${ }^{44}$ and compared with our $4 \mathrm{C}$-Seq data.

Statistical analysis. Data in the graphs are mean \pm s.e.m. of three independent experiments with triplicated samples. $P$ values were assessed using unpaired and two-tailed Student's $t$ test (all figures except Figs. 1e, 2c, and 5d, Supplementary Fig. 3e), Mann-Whitney $U$ test (for Figs. 1e and $5 \mathrm{~d}$ ), and two-tailed Fisher's exact test (Fig. 2c, Supplementary Fig. 3e), with R. ${ }^{\star} P<0.05,{ }^{*} P<0.01,{ }^{* * *} P<0.001$. Values of $P<0.05$ were considered statistically significant.

Reporting summary. Further information on research design is available in the Nature Research Reporting Summary linked to this article.

\section{Data availability}

4C-seq and Hi-C datasets have been deposited in the DNA Data Bank of Japan (DDBJ) Sequence Read Archive with the accession number DRA006154 and DRA007945. RNAseq datasets used are ones we previously published ${ }^{27}$. Hi-C and ChIP-seq datasets used are publicly available $16,29,44$. Other data that support the findings of this study are available from the corresponding author upon reasonable request. The source data underlying Figs. 1c, e, 2c-e, 3b, c, 4b, c, 5a, b, d, e, and 6a-c and Supplementary Figs. 2a, d, e, 3b-f, 4a, b, d-i, 5c-d, 6a, 7a-g, 8a, c, f, g, 9a, b, and 10b are provided as a Source Data file.

Received: 22 June 2018 Accepted: 8 July 2019

Published online: 22 August 2019

\section{References}

1. Ali, S. \& Coombes, R. C. Endocrine-responsive breast cancer and strategies for combating resistance. Nat. Rev. Cancer 2, 101-112 (2002).

2. Yager, J. D. \& Davidson, N. E. Estrogen carcinogenesis in breast cancer. $N$. Engl. J. Med. 354, 270-282 (2006)

3. Musgrove, E. A. \& Sutherland, R. L. Biological determinants of endocrine resistance in breast cancer. Nat. Rev. Cancer 9, 631-643 (2009).

4. Jeng, M. H. et al. Estrogen receptor expression and function in long-term estrogen-deprived human breast cancer cells. Endocrinology 139, 4164-4174 (1998).

5. Katzenellenbogen, B. S., Kendra, K. L., Norman, M. J. \& Berthois, Y. Proliferation, hormonal responsiveness, and estrogen receptor content of MCF-7 human breast cancer cells grown in the short-term and long-term absence of estrogens. Cancer Res. 47, 4355-4360 (1987).

6. Santen, R. J. et al. Long-term estradiol deprivation in breast cancer cells upregulates growth factor signaling and enhances estrogen sensitivity. Endocr. Relat. Cancer 12, S61-S73 (2005)

7. Welshons, W. V. \& Jordan, V. C. Adaptation of estrogen-dependent MCF-7 cells to low estrogen (phenol red-free) culture. Eur. J. Cancer Clin. Oncol. 23, 1935-1939 (1987)

8. Song, R. X.-D., Zhang, Z., Mor, G. \& Santen, R. J. Down-regulation of Bcl-2 enhances estrogen apoptotic action in long-term estradiol-depleted ER+ breast cancer cells. Apoptosis 10, 667-678 (2005). 
9. Song, R. X. D. et al. Effect of long-term estrogen deprivation on apoptotic responses of breast cancer cells to $17 \beta$-estradiol. J. Natl. Cancer Inst. 93, 1714-1723 (2001).

10. Iwase, H. et al. Ethinylestradiol is beneficial for postmenopausal patients with heavily pre-treated metastatic breast cancer after prior aromatase inhibitor treatment: a prospective study. Br. J. Cancer 109, 1537-1542 (2013).

11. Lonning, P. E. et al. High-dose estrogen treatment in postmenopausal breast cancer patients heavily exposed to endocrine therapy. Breast Cancer Res. Treat. 67, 111-116 (2001).

12. Lieberman-Aiden, E. et al. Comprehensive Mapping of Long-Range Interactions Reveals Folding Principles of the Human Genome. Science 326, 289-293 (2009).

13. Fanucchi, S. \& Mhlanga, M. M. Enhancer-derived lncRNAs regulate genome architecture: fact or fiction? Trends Genet. 33, 375-377 (2017).

14. Dixon, J. R. et al. Topological domains in mammalian genomes identified by analysis of chromatin interactions. Nature 485, 376-380 (2012).

15. Nora, E. P. et al. Spatial partitioning of the regulatory landscape of the Xinactivation centre. Nature 485, 381-385 (2012).

16. Rao, S. S. et al. A 3D map of the human genome at kilobase resolution reveals principles of chromatin looping. Cell 159, 1665-1680 (2014).

17. Ponting, C. P. \& Belgard, T. G. Transcribed dark matter: meaning or myth? Hum. Mol. Genet 19, R162-R168 (2010).

18. Seila, A. C. et al. Divergent transcription from active promoters. Science 322, 1849-1851 (2008).

19. Preker, P. et al. RNA exosome depletion reveals transcription upstream of active human promoters. Science 322, 1851-1854 (2008).

20. Core, L. J., Waterfall, J. J. \& Lis, J. T. Nascent RNA sequencing reveals widespread pausing and divergent initiation at human promoters. Science 322, 1845-1848 (2008).

21. Yamamoto, T. \& Saitoh, N. Non-coding RNAs and chromatin domains. Curr. Opin. Cell Biol. 58, 26-33 (2019).

22. Isoda, T. et al. Non-coding Transcription instructs chromatin folding and compartmentalization to dictate enhancer-promoter communication and $\mathrm{T}$ cell fate. Cell 171, 103-119 e118 (2017).

23. Nagano, T. et al. The Air noncoding RNA epigenetically silences transcription by targeting G9a to chromatin. Science 322, 1717-1720 (2008).

24. Santoro, F. et al. Imprinted Igf2r silencing depends on continuous Airn lncRNA expression and is not restricted to a developmental window. Development 140, 1184 (2013).

25. Amaral, P. P. et al. Genomic positional conservation identifies topological anchor point RNAs linked to developmental loci. Genome Biol. 19, 32 (2018).

26. Arun, G., Diermeier, S. D. \& Spector, D. L. Therapeutic targeting of long noncoding RNAs in cancer. Trends Mol. Med. 24, 257-277 (2018).

27. Tomita, S. et al. A cluster of noncoding RNAs activates the ESR1 locus during breast cancer adaptation. Nat. Commun. 6, 6966 (2015).

28. Aguilar, $\mathrm{H}$. et al. Biological reprogramming in acquired resistance to endocrine therapy of breast cancer. Oncogene 29, 6071-6083 (2010).

29. Barutcu, A. R. et al. Chromatin interaction analysis reveals changes in small chromosome and telomere clustering between epithelial and breast cancer cells. Genome Biol. 16, 214 (2015).

30. Skurk, C. et al. The Akt-regulated forkhead transcription factor FOXO3a controls endothelial cell viability through modulation of the caspase-8 inhibitor FLIP. J. Biol. Chem. 279, 1513-1525 (2004)

31. Ekoff, M. et al. The BH3-only protein Puma plays an essential role in cytokine deprivation induced apoptosis of mast cells. Blood 110, 3209-3232 (2007).

32. Myatt, S. S. \& Lam, E. W. The emerging roles of forkhead box (Fox) proteins in cancer. Nat. Rev. Cancer 7, 847-859 (2007).

33. Jiang, Y., Zou, L., Lu, W.-Q., Zhang, Y. \& Shen, A.-G. Foxo3a expression is a prognostic marker in breast cancer. PLoS ONE 8, e70746 (2013).

34. Yamamoto, T. et al. Endocrine therapy-resistant breast cancer model cells are inhibited by soybean glyceollin I through Eleanor non-coding RNA. Sci. Rep. 8, 15202 (2018).

35. Wang, D. et al. Reprogramming transcription by distinct classes of enhancers functionally defined by eRNA. Nature 474, 390-394 (2011).

36. Castles, C. G., Oesterreich, S., Hansen, R. \& Fuqua, S. A. Auto-regulation of the estrogen receptor promoter. J. Steroid Biochem. Mol. Biol. 62, 155-163 (1997).

37. Guo, S. \& Sonenshein, G. E. Forkhead box transcription factor FOXO3a regulates estrogen receptor alpha expression and is repressed by the Her-2/ neu/phosphatidylinositol 3-kinase/Akt signaling pathway. Mol. Cell Biol. 24, $8681-8690$ (2004).

38. Zou, Y. et al. Forkhead box transcription factor FOXO3a suppresses estrogendependent breast cancer cell proliferation and tumorigenesis. Breast Cancer Res. 10, R21 (2008).

39. Fortin, J. P. \& Hansen, K. D. Reconstructing A/B compartments as revealed by $\mathrm{Hi}-\mathrm{C}$ using long-range correlations in epigenetic data. Genome Biol. 16, 180 (2015).
40. Fudenberg, G. et al. Formation of chromosomal domains by loop extrusion. Cell Rep. 15, 2038-2049 (2016).

41. Nora, E. P. et al. Targeted degradation of CTCF decouples local insulation of chromosome domains from genomic compartmentalization. Cell 169, 930-944 e922 (2017).

42. Schwarzer, W. et al. Two independent modes of chromatin organization revealed by cohesin removal. Nature 551, 51-56 (2017).

43. Sofueva, S. et al. Cohesin-mediated interactions organize chromosomal domain architecture. EMBO J. 32, 3119-3129 (2013).

44. Consortium, E. P. An integrated encyclopedia of DNA elements in the human genome. Nature 489, 57-74 (2012).

45. Xu, Z., Wei, G., Chepelev, I., Zhao, K. \& Felsenfeld, G. Mapping of INS promoter interactions reveals its role in long-range regulation of SYT8 transcription. Nat. Struct. Mol. Biol. 18, 372-378 (2011).

46. Hao, N., Palmer, A. C., Ahlgren-Berg, A., Shearwin, K. E. \& Dodd, I. B. The role of repressor kinetics in relief of transcriptional interference between convergent promoters. Nucleic Acids Res. 44, 6625-6638 (2016).

47. Mazo, A., Hodgson, J. W., Petruk, S., Sedkov, Y. \& Brock, H. W. Transcriptional interference: an unexpected layer of complexity in gene regulation. J. Cell Sci. 120, 2755-2761 (2007).

48. Tomita, S. et al. Roles of long noncoding RNAs in chromosome domains. Wiley Interdiscip. Rev. RNA https://doi.org/10.1002/wrna.1384 (2017).

49. Wang, K. C. et al. A long noncoding RNA maintains active chromatin to coordinate homeotic gene expression. Nature 472, 120-124 (2011).

50. Habashy, H. O. et al. FOXO3a nuclear localisation is associated with good prognosis in luminal-like breast cancer. Breast Cancer Res. Treat. 129, 11-21 (2011).

51. Zekas, E. \& Prossnitz, E. R. Estrogen-mediated inactivation of FOXO3a by the G protein-coupled estrogen receptor GPER. BMC Cancer 15, 702 (2015).

52. Queiroz, E. A. et al. Metformin induces apoptosis and cell cycle arrest mediated by oxidative stress, AMPK and FOXO3a in MCF-7 breast cancer cells. PLoS ONE 9, e98207 (2014).

53. Giamas, G. et al. Kinome screening for regulators of the estrogen receptor identifies LMTK3 as a new therapeutic target in breast cancer. Nat. Med 17, 715-719 (2011).

54. Johnson, A. B. \& O'Malley, B. W. ERasing breast cancer resistance through the kinome. Nat. Med. 17, 660 (2011).

55. Morelli, C. et al. Akt2 inhibition enables the forkhead transcription factor FoxO3a to have a repressive role in estrogen receptor alpha transcriptional activity in breast cancer cells. Mol. Cell Biol. 30, 857-870 (2010).

56. Bradner, J. E., Hnisz, D. \& Young, R. A. Transcriptional addiction in cancer Cell 168, 629-643 (2017).

57. Ariazi, E. A. et al. Estrogen induces apoptosis in estrogen deprivation-resistant breast cancer through stress responses as identified by global gene expression across time. Proc. Natl Acad. Sci. USA 108, 18879-18886 (2011).

58. Simonis, M., Kooren, J. \& de Laat, W. An evaluation of 3C-based methods to capture DNA interactions. Nat. Methods 4, 895-901 (2007)

59. Langmead, B. \& Salzberg, S. L. Fast gapped-read alignment with Bowtie 2. Nat Methods 9, 357-359 (2012).

60. Li, H. et al. The Sequence Alignment/Map format and SAMtools. Bioinformatics 25, 2078-2079 (2009).

61. van de Werken, H. J. G. et al. Robust $4 \mathrm{C}$-seq data analysis to screen for regulatory DNA interactions. Nat. Methods 9, 969 (2012).

62. Ikeda, T. et al. Srf destabilizes cellular identity by suppressing cell-type-specific gene expression programs. Nat. Commun. 9, 1387 (2018).

63. Durand, N. C. et al. Juicer provides a one-click system for analyzing loopresolution Hi-C experiments. Cell Syst. 3, 95-98 (2016).

64. Miura, H., Poonperm, R., Takahashi, S. \& Hiratani, I. Practical analysis of HiC data: generating A/B compartment profiles. Methods Mol. Biol. 1861, 221-245 (2018).

65. Stansfield, J. C., Cresswell, K. G., Vladimirov, V. I. \& Dozmorov, M. G. HiCcompare: an R-package for joint normalization and comparison of HI-C datasets. BMC Bioinformatics 19, 279 (2018).

66. Wang, Y. et al. The 3D Genome Browser: a web-based browser for visualizing 3D genome organization and long-range chromatin interactions. Genome Biol. 19, 151 (2018).

67. Richter, A. S. et al. deepTools2: a next generation web server for deepsequencing data analysis. Nucleic Acids Res. 44, W160-W165 (2016).

68. Quinlan, A. R. \& Hall, I. M. BEDTools: a flexible suite of utilities for comparing genomic features. Bioinformatics 26, 841-842 (2010).

69. Heinz, S. et al. Simple combinations of lineage-determining transcription factors prime cis-regulatory elements required for macrophage and B cell identities. Mol. Cell 38, 576-589 (2010).

70. Dacwag, C. S., Ohkawa, Y., Pal, S., Sif, S. \& Imbalzano, A. N. The protein arginine methyltransferase Prmt5 is required for myogenesis because it facilitates ATP-dependent chromatin remodeling. Mol. Cell. Biol. 27, 384-394 (2007).

71. Schneider, C. A., Rasband, W. S. \& Eliceiri, K. W. NIH Image to ImageJ: 25 years of image analysis. Nat. Methods 9, 671-675 (2012). 


\section{Acknowledgements}

We thank all of the members of the Nakao and Saitoh laboratories for discussions and technical assistance. We thank Dr. Gary Felsenfeld (NIH), Dr. Musa Mhlanga (University of Cape Town), and Dr. Yutaka Negishi (University of Cape Town) for critical reading of the manuscript and helpful discussions. We appreciate Dr. Hiroshi Kimura (Tokyo Institute of Technology) for important discussion. We also thank the Advanced Computational Scientific Program of the Research Institute for Information Technology, Kyushu University for providing high-performance computing resources. This work was supported by the JSPS KAKENHI (18H04904, 19H04970 [to K.M.], 18H04802, $18 \mathrm{H} 05527,19 \mathrm{H} 05244$ [to Y.O.], $18 \mathrm{H} 05530$ [to I.H.], 18K19479 [to M.N.], and 18H05531, 18 K19310 [to N.S.]); JST CREST JPMJCR16G1 [to Y.O.]; Mitsubishi Foundation [to M. N.]; AMED CREST JP16gm0510007 [to M.N.]; The Naito Foundation; Takeda Science Foundation; and The Vehicle Racing Commemorative Foundation [to N.S.]. This work was partly performed in the Cooperative Research Project Program of the Medical Institute of Bioregulation, Kyushu University.

\section{Author contributions}

M.O.A.A. and T.Y. performed most of the experiments. K.M., J.N. and Y.O. performed sequencing and data analyses. H.M., R.P. and I.H. analyzed Hi-C data for TAD profiling. N.S., M.N. and Y.O. conceived, designed, and supervised the work. N.S. and T.Y. wrote the paper with H.N. and M.N. All the authors discussed the results and commented on the manuscript.

\section{Additional information}

Supplementary Information accompanies this paper at https://doi.org/10.1038/s41467019-11378-4.
Competing interests: The authors declare no competing interests.

Reprints and permission information is available online at http://npg.nature.com/ reprintsandpermissions/

Peer review information: Nature Communications thanks Erwei Song and other anonymous reviewer(s) for their contribution to the peer review of this work. Peer reviewer reports are available.

Publisher's note: Springer Nature remains neutral with regard to jurisdictional claims in published maps and institutional affiliations.

(c) (i) Open Access This article is licensed under a Creative Commons Attribution 4.0 International License, which permits use, sharing, adaptation, distribution and reproduction in any medium or format, as long as you give appropriate credit to the original author(s) and the source, provide a link to the Creative Commons license, and indicate if changes were made. The images or other third party material in this article are included in the article's Creative Commons license, unless indicated otherwise in a credit line to the material. If material is not included in the article's Creative Commons license and your intended use is not permitted by statutory regulation or exceeds the permitted use, you will need to obtain permission directly from the copyright holder. To view a copy of this license, visit http://creativecommons.org/ licenses/by/4.0/.

(C) The Author(s) 2019 